\title{
PRECONDITIONING THE HELMHOLTZ EQUATION WITH THE SHIFTED LAPLACIAN AND FABER POLYNOMIALS*
}

\author{
LUIS GARCÍA RAMOS ${ }^{\dagger}$, OLIVIER SÈTE ${ }^{\dagger}$, AND REINHARD NABBEN ${ }^{\dagger}$
}

\begin{abstract}
We introduce a new polynomial preconditioner for solving the discretized Helmholtz equation preconditioned with the complex shifted Laplace (CSL) operator. We exploit the localization of the spectrum of the CSL-preconditioned system to approximately enclose the eigenvalues by a non-convex 'bratwurst' set. On this set, we expand the function $1 / z$ into a Faber series. Truncating the series gives a polynomial, which we apply to the Helmholtz matrix preconditioned by the shifted Laplacian to obtain a new preconditioner, the Faber preconditioner. We prove that the Faber preconditioner is nonsingular for degrees one and two of the truncated series. Our numerical experiments (for problems with constant and varying wavenumber) show that the Faber preconditioner reduces the number of GMRES iterations.
\end{abstract}

Key words. Helmholtz equation, shifted Laplace preconditioner, iterative methods, GMRES, preconditioning, Faber polynomials, 'bratwurst' sets

AMS subject classifications. 65F08, 65F10, 30C10, 30C20

1. Introduction. We study the solution of linear systems of equations

$$
A x=b
$$

arising from the discretization of the Helmholtz equation. The Helmholtz (or reduced wave) equation results from the modelling of time-harmonic solutions of the standing wave equation. Such models appear in various branches of science and engineering, for example, in acoustics, seismic exploration, and medical imaging. Discretizing the Helmholtz equation on a domain equipped with boundary conditions by the finite difference or finite element method yields the linear system (1.1) with a matrix $A$ that is complex symmetric, non-Hermitian, and indefinite. In practical applications, the system of equations is too large to be solved by direct methods, and iterative solvers are used instead. However, most classical iterative methods are known to perform poorly when applied to the Helmholtz equation; see the survey [22].

A standard approach is to use Krylov subspace methods in combination with a preconditioner, that is, instead of solving (1.1) directly, one finds a matrix $M^{-1}$ that approximates $A^{-1}$ and solves the system

$$
A M^{-1} y=b
$$

with a Krylov method and computes $x=M^{-1} y$. In the last two decades, many different preconditioners for the Helmholtz problem have been described. Some of the most efficient ones are domain-decomposition-based preconditioners (in particular sweeping-type preconditioners) and the shifted Laplacian. These two techniques are also used in combination; see $[16,24,27,53,59]$ and the survey articles [17, 22, 23] for more references.

Among the latter, a widely used method is the complex shifted Laplacian (CSL) introduced in [20] and further developed in [19, 21]; see also [1, 4, 7, 23, 45]. In [18, 25, 49, 50], multilevel Krylov and multilevel deflation techniques to accelerate the CSL preconditioner are introduced and analyzed. For a survey, see [17], and for an overview of recent developments and applications of the CSL preconditioner, see the book [34]. The CSL preconditioner is

* Received July 17, 2019. Accepted July 16, 2021. Published online on September 6, 2021. Recommended by Bernd Beckermann.

${ }^{\dagger}$ Technische Universität Berlin, Institute of Mathematics, Straße des 17. Juni 136, 10623 Berlin, Germany ( $\{$ garcia, sete, nabben\}@math.tu-berlin.de). 
one of the building blocks of some of the most efficient preconditioners for the Helmholtz equation currently in use; see [34]. In this work, we investigate the combination of the CSL preconditioner with a polynomial preconditioner.

Polynomial preconditioning for the solution of linear systems is a well-known technique; see $[10,35,47]$ and the books $[26,48,57]$. There, the preconditioner is a polynomial in $A$, i.e., $M^{-1}=p(A)$. One idea to obtain such a polynomial is to expand $A^{-1}$ into a convergent polynomial series and use a truncation of the series as preconditioner. The simplest form is a Neumann series expansion. Other approaches construct a polynomial by solving a minimization problem, e.g., Chebyshev polynomials [33] or least-squares polynomials [55], which both require an estimate of the spectrum of the matrix, or polynomials related to the minimum residual polynomial generated by running a few steps of a GMRES iteration [37]. Polynomial preconditioning for the Helmholtz equation was used in [8]. There, the inverse of the Helmholtz operator is expanded into a Taylor series around a complex shifted Laplacian operator, and the resulting truncated series is used as a preconditioner. The preconditioner based on a rational approximation for indefinite linear systems introduced in [58] has also been applied to the Helmholtz equation.

Polynomial preconditioning can also be applied to the preconditioned system, i.e., instead of solving

$$
A M^{-1} x=b,
$$

one solves the polynomially preconditioned system

$$
A M^{-1} p\left(A M^{-1}\right) y=b,
$$

with a suitable polynomial $p$, and computes $x=p\left(A M^{-1}\right) y$. Polynomial approximation techniques can also be combined with multigrid and multilevel methods, e.g., as smoothers for difficult problems where typical smoothers only give slow convergence or even divergence of the multilevel scheme [15]. A polynomial related to the best approximation of $1 / x$ in the supremum norm on an interval has been applied to two-level methods in [33] for the case that the eigenvalues lie inside a real interval.

A general principle underlying many of these approaches is that if a polynomial $p^{*}$ approximates the function $f(z)=1 / z$ on a set that contains the spectrum of $A$ (or of the preconditioned matrix $\left.A M^{-1}\right)$, then $p^{*}(A)$ (respectively, $p^{*}\left(A M^{-1}\right)$ ) can be used as a preconditioner for the linear system. In this work we follow this principle and approximate the function $1 / z$ on a set $E$ containing the spectrum of $A M^{-1}$ by Faber polynomials. Faber polynomials have been used previously in numerical analysis and in numerical linear algebra, e.g., in $[3,11,13,14,29,42,43,52]$.

We exploit the fact that the spectrum of the CSL-preconditioned system $A M^{-1}$ lies in the disk $\{z \in \mathbb{C}:|z-1 / 2| \leq 1 / 2\}$ (see Theorem 2.1), which was first proved in [56], and we approximately enclose the spectrum by a 'bratwurst' set [31]. The function $f(z)=1 / z$ is analytic on our inclusion set $E$ and can be expanded into a convergent Faber series, which we determine explicitly. We then use the truncated Faber series of the preconditioned matrix as a new preconditioner for a Krylov subspace method. In our numerical experiments we truncate the series after at most three terms.

The polynomial preconditioner developed in [8] for the Helmholtz equation reduces the number of iterations but the overall CPU time increases. Our experiments show that our new polynomial preconditioner of the CSL preconditioner reduces the number of iterations and sometimes also the CPU time compared to the use of the CSL preconditioner only.

This paper is organized as follows. In Section 2 we introduce the discrete Helmholtz problem and the shifted Laplacian. The polynomial preconditioner is constructed in Section 3, 
where we also briefly review the theory of Faber series, the definition of 'bratwurst' sets, and the use of these for polynomial preconditioning. Section 4 presents the results of numerical experiments for one- and two-dimensional Helmholtz problems, and in Section 5 we present some conclusions.

2. The Helmholtz equation and the complex shifted Laplace preconditioner. In this section we discuss the discretization of the Helmholtz equation and the complex shifted Laplace operator. For a bounded domain $\Omega \subset \mathbb{R}^{d}$ and $k \in \mathbb{R}$, the Helmholtz equation is given by

$$
-\Delta u-k^{2} u=f
$$

with Dirichlet boundary conditions of the form

$$
u=0 \quad \text { on } \quad \partial \Omega,
$$

or first-order absorbing boundary conditions (sometimes also called impedance boundary conditions, e.g., in [23], or Sommerfeld condition, e.g., in [20])

$$
\partial_{n} u-i k u=0 \quad \text { on } \quad \partial \Omega .
$$

The parameter $k$ is the wavenumber. In many applications the dimension $d$ is equal to 2 or 3 , and the wavenumber can be large. After discretization by first-order finite differences on a grid of meshsize $h$ or by linear finite elements, we obtain a discrete Helmholtz equation of the form

$$
A x=b,
$$

where

$$
A=L-k^{2} S-i k C,
$$

in which $L$ is the discretization of the Laplacian, $C$ arises from the discretization of the absorbing boundary conditions (so $C=0$ in case of Dirichlet boundary conditions), and $S$ results from the zeroth-order term [56]. The matrices $L$ and $S$ are real, symmetric, and positive definite, and $C$ is positive semidefinite. Since the solutions to (2.1) are oscillatory, the number of gridpoints in one dimension must scale linearly with $k$. Therefore, the gridsize is chosen by requiring a fixed number of points per wavelength, typically at least 10 [2], or equivalently, keeping the quantity $k h$ constant as $k$ increases. This rule, however, is not sufficient to maintain the accuracy of the solutions at very high wavenumbers due to the 'pollution effect' caused by numerical dispersion [30]. It has been shown that for high wavenumbers, the gridsize should scale at least linearly with $k^{3 / 2}$ [41]. For some applications, this leads to very large linear systems of equations.

For $\varepsilon \in \mathbb{R}$, the complex shifted Laplacian preconditioner (CSL preconditioner) corresponds to the discretization of the problem

$$
-\Delta u-\left(k^{2}+i \varepsilon\right) u=f
$$

with the same boundary conditions as the original Helmholtz problem. The choice of the boundary conditions can be justified by the results in [23,39]. This results in the discrete problem $A_{\varepsilon} x=b$ with the coefficient matrix

$$
A_{\varepsilon}=L+i k C-\left(k^{2}+i \varepsilon\right) S=A-i \varepsilon S .
$$




\section{ETNA}

Kent State University and Johann Radon Institute (RICAM)
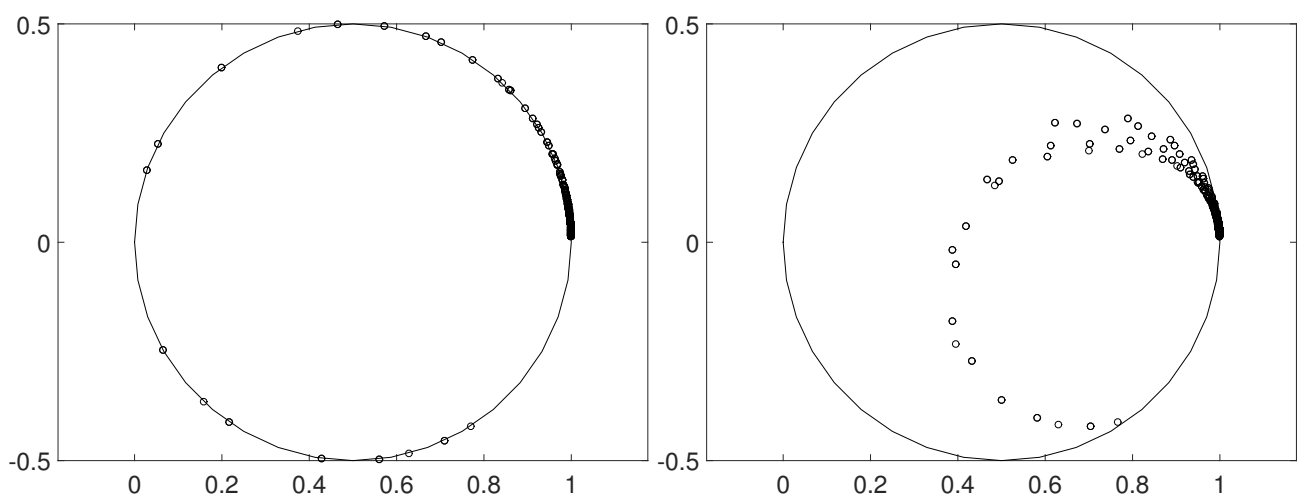

FIG. 2.1. The spectrum of $A A_{\varepsilon}^{-1}$ with Dirichlet (left) and absorbing (right) boundary conditions on $\Omega=[0,1]^{2}$ with parameters $k=15, \beta=0.5$.

The matrix $A_{\varepsilon}$ is used as a preconditioner for (2.2). The resulting right-preconditioned problem is

$$
A A_{\varepsilon}^{-1} y=b, \quad x=A_{\varepsilon}^{-1} y .
$$

The CSL problem in (2.4) can be interpreted physically as an artificially damped Helmholtz problem, where the damping allows the preconditioner $A_{\varepsilon}$ to be inverted efficiently, for example, by standard multigrid methods. The shift should be chosen to minimize the number of iterations required to solve the preconditioned system, while still being able to (approximately) solve the shifted system. It has been shown in [23] that if the GMRES method is used for the outer Krylov iteration, then a sufficient condition to ensure a number of iterations independent of the wavenumber is that the shift scales linearly with respect to $k$, that is, $\varepsilon=O(k)$. This, however, poses difficulties for the inversion of the preconditioner $A_{\varepsilon}$, in particular when multigrid methods are used. Indeed, it was shown in [5] that the shift $\varepsilon$ must be at least $O\left(k^{2}\right)$ for the shifted Laplace preconditioner to be able to be inverted using multigrid methods for high wavenumbers, and earlier publications indicate that the choice $\varepsilon=k^{2} \beta$ for a fixed $\beta \in(0,1)$ can lead to an efficient preconditioner for moderate wavenumbers when $A_{\varepsilon}$ is inverted with the multigrid method [19]. This has been further justified by a spectral analysis and local Fourier analysis in $[7,56]$, where the authors found that $\beta=0.5$ leads to a nearly optimal method in terms of Krylov iterations. We use this choice in our numerical experiments.

The eigenvalues of the preconditioned matrix $A A_{\varepsilon}^{-1}$ lie in a disk. This was first proved in [56]; see also [25] for an alternative proof. We use this localization of the spectrum of $A A_{\varepsilon}^{-1}$ to develop our polynomial preconditioner.

THEOREM 2.1. In dimension $d=1,2$, or 3 , let $A$ and $A_{\varepsilon}$ be given by (2.3) and (2.5), respectively, and let $\varepsilon=k^{2} \beta$ with $\beta>0$. Then the spectrum of the preconditioned matrix $A A_{\varepsilon}^{-1}$ lies in the closed disk

$$
\{z \in \mathbb{C}:|z-1 / 2| \leq 1 / 2\}
$$

In case of Dirichlet boundary conditions, the spectrum of $A A_{\varepsilon}^{-1}$ lies on the boundary of (2.6).

Theorem 2.1 is illustrated in Figure 2.1 for Helmholtz problems on $\Omega=[0,1] \times[0,1]$ with $k=15$ and $\beta=0.5$.

3. Construction of the Faber preconditioner from the Faber series. In order to construct a polynomial preconditioner for solving the CSL-preconditioned Helmholtz equation 


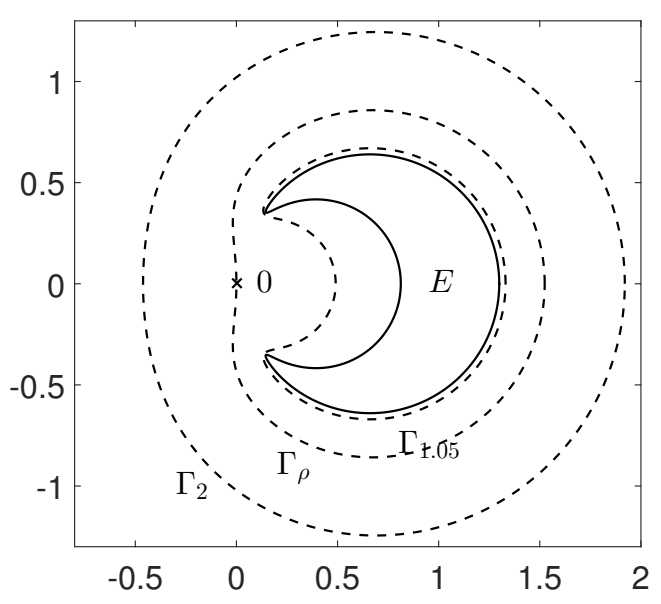

FIG. 3.1. The set $E$ and several contour lines $\Gamma_{r}$.

$A A_{\varepsilon}^{-1} x=b$ by GMRES, we approximately enclose the spectrum of $A A_{\varepsilon}^{-1}$ by a non-convex set and expand $1 / z$ into a Faber series on the inclusion set. The truncated series is a polynomial. Applying it to $A A_{\varepsilon}^{-1}$ gives the Faber preconditioner.

3.1. Faber polynomials and the Faber series. Faber polynomials allow the series expansion of analytic functions on certain compact subsets of $\mathbb{C}$. In this section, we summarize the definition and some properties of the Faber polynomials and the Faber series, which can be found alongside with further properties in, e.g., [9], [54, Ch. III], [40, Sect. 14], or [51, Ch. 2].

Let $E \subset \mathbb{C}$ be a compact set (not a single point) with simply connected complement $E^{c}:=\widehat{\mathbb{C}} \backslash E$ in the extended complex plane $\widehat{\mathbb{C}}=\mathbb{C} \cup\{\infty\}$. By the Riemann mapping theorem there exists a unique conformal map

$$
\psi:\{w \in \widehat{\mathbb{C}}:|w|>1\} \rightarrow E^{c} \quad \text { with } \quad \psi(\infty)=\infty, \quad \psi^{\prime}(\infty)>0 .
$$

We denote the image of the circle $|w|=r$ under $\psi$ by

$$
\Gamma_{r}=\psi(\{w \in \mathbb{C}:|w|=r\}), \quad r>1 .
$$

The curves $\Gamma_{r}$ are the level curves of the Green function for $E^{c}$ with the pole at infinity, given by $g_{E^{c}}(z)=\log \left|\psi^{-1}(z)\right|$. Figure 3.1 displays a compact set $E$ (bounded by the solid line) and several curves $\Gamma_{r}$ (the dashed lines).

For a fixed $z \in \mathbb{C}$, the function $\psi^{\prime}(w) /(\psi(w)-z)$ is analytic in a neighbourhood of infinity and can thus be expanded into a Laurent series

$$
\frac{\psi^{\prime}(w)}{\psi(w)-z}=\sum_{k=0}^{\infty} \frac{F_{k}(z)}{w^{k+1}}, \quad z \text { interior to } \Gamma_{r}, \quad|w|>r .
$$

Each coefficient $F_{k}(z)$ is a polynomial of degree $k$, the $k$-th Faber polynomial of $E$. The leading coefficient of $F_{k}(z)$ is $\left(\psi^{\prime}(\infty)\right)^{-k}$, where $\psi^{\prime}(\infty)=c(E)$ is the logarithmic capacity of $E$.

Let $f$ be an analytic function on the compact set $E$, that is, $f$ is analytic in an open set containing $E$. Therefore, there exists a largest $\rho>1$ such that $f$ is analytic in the interior of the curve $\Gamma_{\rho}$ but not in the interior of a larger curve (for an entire function, $\rho=\infty$ ). The 
function $f$ has a unique representation as a Faber series

$$
f(z)=\sum_{k=0}^{\infty} a_{k} F_{k}(z)
$$

which converges absolutely in the interior of $\Gamma_{\rho}$, uniformly on compact sets contained in the interior of $\Gamma_{\rho}$, and diverges in the exterior of $\Gamma_{\rho}$. The coefficients are given by

$$
a_{k}=\frac{1}{2 \pi i} \int_{|w|=r} \frac{f(\psi(w))}{w^{k+1}} d w
$$

where $1<r<\rho$ is arbitrary. Here and in the sequel we integrate over the circle oriented as usual in the positive sense (counter-clockwise). In our application, we compute the Faber polynomials and the coefficients analytically.

EXAMPLE 3.1.

1. For the disk $E=\{z \in \mathbb{C}:|z| \leq 1\}$, we have $\psi(z)=z, F_{k}(z)=z^{k}$, and (3.2) is a Taylor series with radius of convergence $\rho$.

2. For the interval $E=[-1,1]$, we have $\psi(z)=\frac{1}{2}\left(z+z^{-1}\right)$, and the Faber polynomials are related to the Chebyshev polynomials of the first kind by $F_{0}(z)=T_{0}(z)$ and $F_{k}(z)=2 T_{k}(z)$, for $k \geq 1$.

The Faber series (3.2) converges geometrically to $f$ on $E$, which is the fastest possible convergence in an $n$-th root sense. This is expressed by

$$
\limsup _{n \rightarrow \infty}\left\|f-\sum_{k=0}^{n} a_{k} F_{k}\right\|_{E}^{1 / n}=\frac{1}{\rho} ;
$$

see [28]. Here $\|\cdot\|_{E}$ denotes the supremum norm on $E$. Estimates for $\left\|f-\sum_{k=0}^{n} a_{k} F_{k}\right\|_{E}$ for fixed $n$ are given, e.g., in [14, 32], [51, p. 142], and [54, Ch. 8 and 9]. We give an error estimate for our case in Section 3.3.

Faber series can also be used to define matrix functions as follows. Let $A$ be a square matrix whose eigenvalues lie in $E$. Inserting $A$ in the scalar Faber series (3.2) yields

$$
f(A)=\sum_{k=0}^{\infty} a_{k} F_{k}(A),
$$

and this series of matrices converges in any matrix norm $\|\cdot\|$ with

$$
\limsup _{n \rightarrow \infty}\left\|f(A)-\sum_{k=0}^{n} a_{k} F_{k}(A)\right\|^{1 / n} \leq \frac{1}{\rho} ;
$$

see [28, p. 316] or [42], where functions of matrices are computed by (3.4). If the spectrum of $A$ is contained in the interior of $\Gamma_{\rho}$ but not necessarily in $E$, then the series still converges but at a lower rate.

3.2. The inclusion set. We construct a set containing (most of) the spectrum of the CSL-preconditioned Helmholtz operator $A A_{\varepsilon}^{-1}$ (see Figure 2.1) and excluding the origin. The inclusion set is a scaled and shifted variant of the 'bratwurst' sets introduced in [31] and has the form depicted in Figure 3.1. The 'bratwurst' sets are defined through a conformal map 

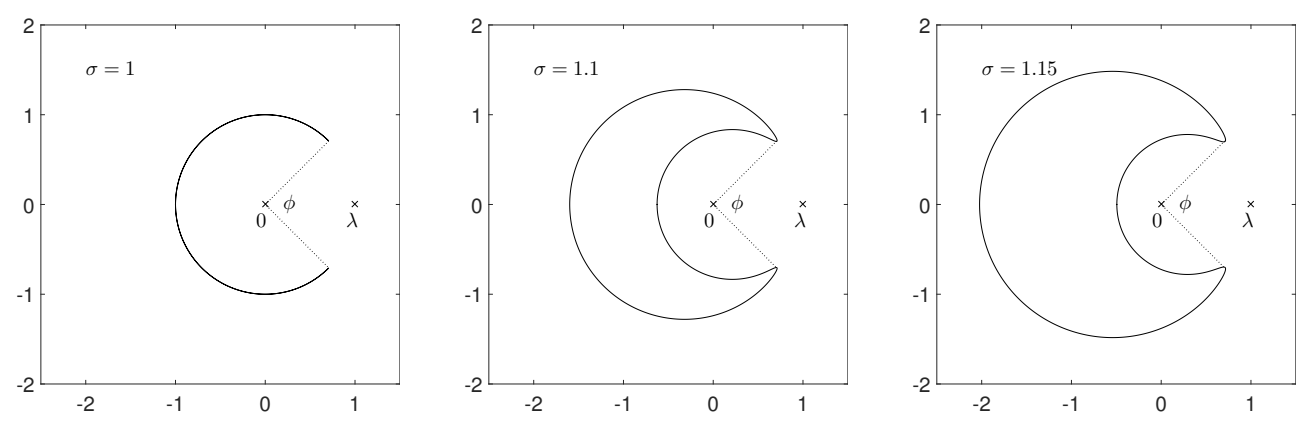

FIG. 3.2. The sets $\Omega(1, \pi / 2, \sigma)$ for $\sigma=1$ (arc), $\sigma=1.1$, and $\sigma=1.15$.

from the exterior of the unit disk onto the exterior of the set. The following theorem describes their construction.

TheOREM 3.2 ([31, Theorem 3.1]). Let $\lambda \in \mathbb{C}$ with $|\lambda|=1, \phi \in] 0,2 \pi[$, and $\sigma \in[1, P[$, where $P=\tan (\phi / 4)+\frac{1}{\cos (\phi / 4)}$, and let

$$
\widetilde{\psi}(w)=\frac{(w-\lambda N)(w-\lambda M)}{(N-M) w+\lambda(N M-1)},
$$

where $M=\frac{\sigma^{2}-1}{2 \sigma \tan (\phi / 4)}$ and $N=\frac{1}{2}\left(\frac{P}{\sigma}+\frac{\sigma}{P}\right)$. Define $\Omega=\Omega(\lambda, \phi, \sigma)$ to be the compact set bounded by $\widetilde{\psi}(\{w \in \mathbb{C}:|w|=1\})$. Then $\widetilde{\psi}$ is a bijective conformal map from the exterior of the unit circle onto $\widehat{\mathbb{C}} \backslash \Omega$ that satisfies $\widetilde{\psi}(\infty)=\infty$ and $\psi^{\prime}(\infty)>0$. We further have $\lambda \notin \Omega$ and $\left\{\lambda e^{i \beta}: \phi / 2 \leq \beta \leq 2 \pi-\phi / 2\right\} \subseteq \Omega$.

The constants $M$ and $N$ satisfy $0 \leq M<1<N$; see [31, Eq. (3.16)].

Figure 3.2 displays the sets $\Omega(1, \pi / 2, \sigma)$ for different $\sigma$. The parameter $\lambda$ indicates where the "opening" is, $\phi$ is related to the width of the opening, and $\sigma$ to how "thick" $\Omega$ is. For $\sigma=1$ it is a Jordan arc [31, Example 3.2] and for $1<\sigma<P$ a set bounded by an analytic Jordan curve.

The eigenvalues of $A A_{\varepsilon}^{-1}$ lie in the disk $\left\{z \in \mathbb{C}:\left|z-\frac{1}{2}\right| \leq \frac{1}{2}\right\}$. We require an inclusion set of the eigenvalues that excludes the origin, which is the pole of $1 / z$. Let

$$
E=\frac{1}{2} \Omega(-1, \phi, \sigma)+\frac{1}{2}
$$

The choice $\lambda=-1$ implies that $-1 \notin \Omega(-1, \phi, \sigma)$, and thus $0 \notin E$. The parameters $\phi$ and $\sigma$ will be chosen in Section 3.7. The function

$$
\psi(w)=\frac{\widetilde{\psi}(w)+1}{2}=\frac{w^{2}+2 N w+1}{2(N-M) w+2(1-M N)}
$$

is the exterior mapping function of $E$ in (3.5), i.e., $\psi$ is conformal and satisfies (3.1).

3.3. The Faber series of $1 / z$ on the inclusion set. The Faber polynomials for the set $E$ in (3.5) can be computed efficiently by a three-term recurrence; see [36, Section 3]. This follows from the fact that $\psi$ in (3.6) is a rational exterior mapping function of type $(2,1)$. The Faber polynomials of $E$ are given by $F_{0}(z)=1$ and

$$
F_{n}(z)=\widehat{F}_{n}(z)-\left(\frac{M N-1}{N-M}\right)^{n}, \quad n \geq 1,
$$


where the shifted Faber polynomials $\widehat{F}_{n}$ satisfy the three-term recurrence

$$
\begin{aligned}
& \widehat{F}_{0}(z)=2 \\
& \widehat{F}_{1}(z)=2(N-M) z-2 N, \\
& \widehat{F}_{n}(z)=(2(N-M) z-2 N) \widehat{F}_{n-1}(z)+(2(1-M N) z-1) \widehat{F}_{n-2}(z), \quad n \geq 2 .
\end{aligned}
$$

In particular, the leading coefficient of $F_{n}(z)$ is $(2(N-M))^{n}$.

Since $f(z)=1 / z$ is analytic on $E$ (recall that $0 \notin E$ ), it has a convergent Faber series, whose coefficients can be computed explicitly. Figure 3.1 displays $E$ and the contour $\Gamma_{\rho}$ from the next theorem.

THEOREM 3.3. With the notation of Theorem 3.2, the function $f(z)=1 / z$ is analytic on the set $E=\frac{1}{2} \Omega(-1, \phi, \sigma)+\frac{1}{2}$. Its Faber series

$$
f(z)=\frac{1}{z}=\sum_{k=0}^{\infty} a_{k} F_{k}(z)
$$

converges absolutely and locally uniformly interior to $\Gamma_{\rho}$ and diverges exterior to $\Gamma_{\rho}$, where $\rho=N+\sqrt{N^{2}-1}>1$. The coefficients are given by

$$
a_{k}=(-1)^{k} \frac{\rho-M}{\rho^{k+1}} .
$$

Proof. The existence and convergence properties of the Faber series of $f$ follow from the general theory of Faber series; see Section 3.1. It remains to determine the number $\rho$ and to show that the coefficients are given by (3.9).

Since $f$ is analytic in $\mathbb{C}$ except at $z=0$, where it has a pole, the number $\rho$ is determined by $0 \in \Gamma_{\rho}$, i.e., $\rho=\left|\psi^{-1}(0)\right|>1$, where $\psi$ is the conformal map from (3.6). The zeros of $\psi$ are

$$
\alpha:=-N-\sqrt{N^{2}-1} \quad \text { and } \quad \beta:=-N+\sqrt{N^{2}-1} .
$$

In particular, $\alpha+\beta=-2 N$ and $\alpha \beta=1$. Since $N>1$, we find

$$
\alpha=-N-\sqrt{N^{2}-1}<-1<\beta=-N+\sqrt{N^{2}-1}<0 .
$$

Since $\psi$ is defined in $|w|>1$, we have $\psi^{-1}(0)=-N-\sqrt{N^{2}-1}$ and

$$
\rho=\left|\psi^{-1}(0)\right|=N+\sqrt{N^{2}-1}>1 .
$$

Next, we compute the coefficients $a_{k}$. By (3.3), they are given by

$$
a_{k}=\frac{1}{2 \pi i} \int_{|t|=r} \frac{1}{\psi(t)} \frac{1}{t^{k+1}} d t
$$

for any $1<r<\rho$. The integrand is rational with poles at $0, \alpha$, and $\beta$, and we compute the integral (3.11) by the residue theorem. Note that $\alpha$ lies exterior to $|t|=r$ since $|\alpha|=\rho>r$, and $\beta$ lies interior to $|t|=r$ since $|\beta|<1<r$. The residue at the simple pole at $t=\beta$ is given by

$$
\begin{aligned}
\operatorname{res}\left(\frac{1}{\psi(t)} \frac{1}{t^{k+1}}, \beta\right) & =\lim _{t \rightarrow \beta} \frac{t-\beta}{\psi(t) t^{k+1}}=\frac{2(N-M) \beta+2(1-M N)}{\beta-\alpha} \frac{1}{\beta^{k+1}} \\
& =\frac{N-M-\sqrt{N^{2}-1}}{\beta^{k+1}}=-\frac{M+\beta}{\beta^{k+1}},
\end{aligned}
$$


where we inserted the values of $\alpha$ and $\beta$ from (3.10). To compute the residue at $t=0$, which is a pole of order $k+1$, we consider the partial fraction decomposition

$$
\frac{1}{\psi(t)}=\frac{2(N-M) t+2(1-M N)}{t^{2}+2 N t+1}=\frac{M+\alpha}{\alpha-t}+\frac{M+\beta}{\beta-t},
$$

with $\alpha$ and $\beta$ from (3.10). For sufficiently small $|t|$ we have

$$
\frac{1}{\psi(t)}=\frac{M+\alpha}{\alpha} \frac{1}{1-t / \alpha}+\frac{M+\beta}{\beta} \frac{1}{1-t / \beta}=\frac{M+\alpha}{\alpha} \sum_{j=0}^{\infty} \frac{t^{j}}{\alpha^{j}}+\frac{M+\beta}{\beta} \sum_{j=0}^{\infty} \frac{t^{j}}{\beta^{j}},
$$

so that

$$
\operatorname{res}\left(\frac{1}{\psi(t)} \frac{1}{t^{k+1}}, 0\right)=\frac{M+\alpha}{\alpha^{k+1}}+\frac{M+\beta}{\beta^{k+1}} .
$$

Together, the residue theorem yields for (3.11)

$$
a_{k}=\frac{M+\alpha}{\alpha^{k+1}}=(-1)^{k} \frac{N+\sqrt{N^{2}-1}-M}{\left(N+\sqrt{N^{2}-1}\right)^{k+1}}, \quad k \geq 0,
$$

which concludes the proof.

The quantity $\frac{1}{\left|\psi^{-1}(0)\right|}=\frac{1}{\rho}$ is known as the asymptotic convergence factor for polynomials from $\mathcal{P}_{n}(0)=\{p: p$ polynomial with $\operatorname{deg}(p) \leq n, p(0)=1\}$ associated with $E$; see $[12,13]$ and [31, Eq. (2.6)].

3.4. Rate of convergence of the Faber series. How well does the truncated Faber series approximate $1 / z$ in Theorem 3.3? Let $s_{n}=\sum_{k=0}^{n} a_{k} F_{k}$ be a partial sum of the Faber series (3.8). Then

$$
\left\|f-s_{n}\right\|_{E}=\left\|\sum_{k=n+1}^{\infty} a_{k} F_{k}\right\|_{E} \leq \sum_{k=n+1}^{\infty}\left|a_{k}\right|\left\|F_{k}\right\|_{E} .
$$

We have $\left|a_{k}\right|=(\rho-M) / \rho^{k+1}$ from (3.9) and $\left\|F_{k}\right\|_{E} \leq V / \pi$, where $V$ is the boundary rotation of $E$; see [54, p. 182]. We give the definition of the boundary rotation and discuss its computation in Appendix A. The approximation error thus satisfies

$$
\left\|f-s_{n}\right\|_{E} \leq \sum_{k=n+1}^{\infty} \frac{\rho-M}{\rho^{k+1}} \frac{V}{\pi}=\frac{V}{\pi} \frac{\rho-M}{\rho-1} \frac{1}{\rho^{n+1}} .
$$

If the origin (the pole of $1 / z$ ) is close to $E$, then we expect that $\rho>1$ is close to 1 . For instance, for $E=\frac{1}{2} \Omega(-1, \pi / 2,1.1)+\frac{1}{2}$, we have $\left\|f-s_{n}\right\|_{E} \leq 14.31 / 1.36^{n+1}$.

In theory, we can solve the CSL-preconditioned Helmholtz equation

$$
A A_{\varepsilon}^{-1} x=b
$$

by computing

$$
x=\left(A A_{\varepsilon}^{-1}\right)^{-1} b=f\left(A A_{\varepsilon}^{-1}\right) b=\sum_{k=0}^{\infty} a_{k} F_{k}\left(A A_{\varepsilon}^{-1}\right) b
$$

with the Faber series (3.8) of $1 / z$. The series of matrices converges provided that the spectrum of $A A_{\varepsilon}^{-1}$ is contained in $E$ (or in the interior of the curve $\Gamma_{\rho}$ ). For practical purposes, however, 
the rate of convergence of the series is too low. This is due to the expected slow convergence of the scalar Faber series and also to some eigenvalues that are close to the origin, which further decreases the rate of convergence of the series. Therefore, instead of solving (3.12) directly with the Faber series, we take a low-degree partial sum of the Faber series as a polynomial preconditioner for GMRES.

3.5. The Faber preconditioner. We construct our polynomial preconditioner for solving (3.12) with GMRES by truncating the Faber series of $1 / z$. More precisely, we consider the polynomially preconditioned system

$$
A A_{\varepsilon}^{-1} s_{n}\left(A A_{\varepsilon}^{-1}\right) x=b,
$$

where the polynomial $s_{n}(z)=\sum_{k=0}^{n} a_{k} F_{k}(z)$ is the $n$-th partial sum of the Faber series (3.8) of $1 / z$, and $n \geq 1$. We call the polynomial preconditioner $s_{n}\left(A A_{\varepsilon}^{-1}\right)$ the Faber preconditioner of degree $n$, denoted by $\operatorname{FP}(n)$.

When a Krylov method is used to solve (3.13), every iteration requires $\operatorname{deg}\left(s_{n}\right)+1=n+1$ matrix-vector products with $A A_{\varepsilon}^{-1}$, that is, $n+1$ approximate inversions of $A_{\varepsilon}$. This limits our choice to small degrees $n$, and in our experiments we consider $n \leq 3$.

We also have to ensure that the Faber preconditioner $s_{n}\left(A A_{\varepsilon}^{-1}\right)$ is nonsingular. In the limit $n \rightarrow \infty$ we have

$$
\lim _{n \rightarrow \infty} s_{n}\left(A A_{\varepsilon}^{-1}\right)=f\left(A A_{\varepsilon}^{-1}\right)=\left(A A_{\varepsilon}^{-1}\right)^{-1},
$$

which guarantees that $s_{n}\left(A A_{\varepsilon}^{-1}\right)$ is nonsingular for sufficiently large $n$. To show that the Faber preconditioner is nonsingular for small $n$ requires more specific information on the location of the zeros of the polynomials $s_{n}$. We investigate this issue for $n=1,2$ in the following proposition. The proof is postponed to Appendix B.

PROPOSITION 3.4. Let $E=\frac{1}{2} \Omega(-1, \phi, \sigma)+\frac{1}{2}$ be a scaled and shifted 'bratwurst' set as in Theorem 3.2, and let $s_{n}(z)$ be the truncated Faber series of $1 / z$ of degree $n$. If $A A_{\varepsilon}^{-1}$ is a Helmholtz matrix preconditioned by the complex shifted Laplacian and $n=1,2$, then the Faber preconditioner $s_{n}\left(A A_{\varepsilon}^{-1}\right)$ is nonsingular.

3.6. Evaluation of the Faber preconditioner. In our numerical experiments, we compute $s_{n}(B) v$ for $B=A A_{\varepsilon}^{-1}$ and a vector $v$ by using the three-term recurrence of the shifted Faber polynomials. We have

$$
s_{n}(z)=\sum_{k=0}^{n} a_{k} F_{k}(z)=\sum_{k=0}^{n} a_{k} \widehat{F}_{j}(z)-\sum_{k=0}^{n} a_{k}\left(\frac{M N-1}{N-M}\right)^{k} .
$$

Thus, $s_{n}(z)$ can be computed iteratively by $\widehat{s}_{0}(z)$, which collects all constant terms, and $\widehat{s}_{m}(z)=\widehat{s}_{m-1}(z)+a_{m} \widehat{F}_{m}(z)$, which yields $\widehat{s}_{n}(z)=s_{n}(z)$. In the process, the shifted Faber polynomials are computed with their three-term recurrence (see Section 3.3). Inserting the matrix and vector gives a short recurrence for computing $s_{n}\left(A A_{\varepsilon}^{-1}\right) v$.

3.7. Determining parameters for the inclusion set. To determine suitable parameters $\phi$ and $\sigma$ of the inclusion set (3.5), we compared the number of iterations and the computation time to solve

$$
A A_{\varepsilon}^{-1} s_{2}\left(A A_{\varepsilon}^{-1}\right) x=b
$$

with (full) GMRES for the angles $\phi=0.1 \pi, 0.2 \pi, \ldots, 0.9 \pi$ and the corresponding values $\sigma=1: 0.05: P(\phi)$ in the model problem 1 below. The number of iterations and times were the 
smallest for $\sigma$ close to 1 . The effect of the angle seems to be less important. We therefore use $\phi=\frac{\pi}{2}$ and $\sigma=1.005$ in our numerical experiments.

We give a first heuristic explanation. The rate of convergence of the Faber series of $1 / z$ on $E$ depends on the number $\rho$, which measures the distance of $E$ to $z=0$, i.e., the singularity of $1 / z$, with the level curves $\Gamma_{r}$; recall Section 3.1. For fixed $\phi$ this distance is the largest for the arc and decreases as $\sigma$ increases. Thus considering for $E$ just an arc gives the fastest approximation of $1 / z$ on $E$. Note that the Faber series also converges interior to other level curves $\Gamma_{r}, 1<r<\rho$, with the convergence being slower for larger $r$. Thus, even if the spectrum of the matrix $A A_{\varepsilon}^{-1}$ is not contained in $E$ but only in the interior of some level curve $\Gamma_{r}$ with small $r$, we will have a high rate of convergence. A rigorous explanation for the matrix case is, however, subject to further work.

4. Numerical experiments. We present the results of numerical experiments for solving Helmholtz problems preconditioned with the complex shifted Laplacian in combination with Faber preconditioners. We consider four model problems: The Helmholtz equation

1. on $(0,1)$ with Dirichlet boundary conditions,

2. on $(0,1)$ with absorbing boundary conditions,

3. on $(0,1)^{2}$ with absorbing boundary conditions,

4. on $(0,1)^{2}$ with absorbing boundary conditions and variable wavenumber.

We solve the linear system of algebraic equations

$$
A A_{\varepsilon}^{-1} x=b
$$

using the Faber preconditioner as described in Section 3.5, i.e., we solve

$$
A A_{\varepsilon}^{-1} s_{n}\left(A A_{\varepsilon}^{-1}\right) y=b
$$

with full or restarted GMRES, and then compute $x=s_{n}\left(A A_{\varepsilon}^{-1}\right) y$. Here $A_{\varepsilon}^{-1}$ is the complex shifted Laplace preconditioner with $\varepsilon=0.5 k^{2}$ (see Section 2), and $s_{n}$ is the truncated Faber series of $1 / z$ of degree $n$ on the inclusion set (3.5) with parameters $\phi=\pi / 2$ and $\sigma=1.005$. Then the Faber preconditioners $s_{1}\left(A A_{\varepsilon}^{-1}\right)$ and $s_{2}\left(A A_{\varepsilon}^{-1}\right)$ are nonsingular (Proposition 3.4), and also $s_{3}\left(A A_{\varepsilon}^{-1}\right)$ is nonsingular since the numerically computed roots of $s_{3}$ lie outside the disk $\{z \in \mathbb{C}:|z-1 / 2| \leq 1 / 2\}$. For comparison, we also solve the system (4.1) preconditioned with the CSL but without the Faber preconditioner.

In our experiments, we consider two variants to invert the preconditioner $A_{\varepsilon}^{-1}$ : via a precomputed LU decomposition in the method denoted by CSL(LU) (only for 1D problems and for reference, since this is not feasible for practical problems) and with a $V(1,1)$ (or $F(1,1)$ for 2D problems) multigrid cycle with $\omega$-Jacobi as a smoother (with relaxation parameter $\omega=2 / 3$ ) in the method CSL(MG). The methods FP( $n)+\mathrm{CSL}(\mathrm{LU})$, and FP( $n)+\mathrm{CSL}(\mathrm{MG})$ result from combining the respective variant of the shifted Laplacian with a Faber preconditioner of degree $n$. Since the preconditioner is relatively expensive to compute, we only use low degrees $n=1,2,3$.

All experiments have been performed in MATLAB 2018b on a Laptop with an i7 processor and 16 GB RAM. We use our own discretization of the Helmholtz equation and multigrid implementation.

4.1. Model problem 1. We consider a Helmholtz problem in one dimension with Dirichlet boundary conditions:

$$
\begin{aligned}
-u^{\prime \prime}-k^{2} u & =f \quad \text { in }(0,1), \\
u(0)=u(1) & =0
\end{aligned}
$$




\section{ETNA}

Kent State University and Johann Radon Institute (RICAM)

TABLE 4.1

Model problem 1: Number of GMRES iterations, matrix-vector products, and timings for computing the shifted Laplacian with an LU factorization.

\begin{tabular}{ccccccccccccc}
$k$ & \multicolumn{4}{c}{ CSL(LU) } & \multicolumn{3}{c}{$\mathrm{FP}(1)+\mathrm{CSL}(\mathrm{LU})$} & \multicolumn{3}{c}{$\mathrm{FP}(2)+\mathrm{CSL}(\mathrm{LU})$} & \multicolumn{3}{c}{$\mathrm{FP}(3)+\mathrm{CSL}(\mathrm{LU})$} \\
& Iter. & MV & Time(s) & Iter. & MV & Time(s) & Iter. & MV & Time(s) & Iter. & MV & Time(s) \\
\hline 20 & 16 & 16 & 0.059 & 17 & 34 & 0.020 & 13 & 39 & 0.045 & 11 & 44 & 0.048 \\
40 & 25 & 25 & 0.037 & 22 & 44 & 0.012 & 16 & 48 & 0.045 & 14 & 56 & 0.046 \\
60 & 32 & 32 & 0.044 & 26 & 52 & 0.026 & 19 & 57 & 0.050 & 16 & 64 & 0.050 \\
80 & 38 & 38 & 0.045 & 28 & 56 & 0.029 & 21 & 63 & 0.052 & 17 & 68 & 0.050 \\
100 & 47 & 47 & 0.053 & 33 & 66 & 0.022 & 25 & 75 & 0.058 & 21 & 84 & 0.054 \\
120 & 51 & 51 & 0.068 & 35 & 70 & 0.034 & 25 & 75 & 0.060 & 21 & 84 & 0.058 \\
150 & 61 & 61 & 0.078 & 40 & 80 & 0.042 & 30 & 90 & 0.078 & 25 & 100 & 0.062 \\
200 & 76 & 76 & 0.112 & 49 & 98 & 0.040 & 36 & 108 & 0.088 & 29 & 116 & 0.078 \\
400 & 131 & 131 & 0.309 & 79 & 158 & 0.153 & 57 & 171 & 0.132 & 47 & 188 & 0.133 \\
600 & 182 & 182 & 0.717 & 107 & 214 & 0.300 & 78 & 234 & 0.290 & 63 & 252 & 0.247 \\
800 & 236 & 236 & 1.120 & 137 & 274 & 0.412 & 100 & 300 & 0.395 & 81 & 324 & 0.333
\end{tabular}
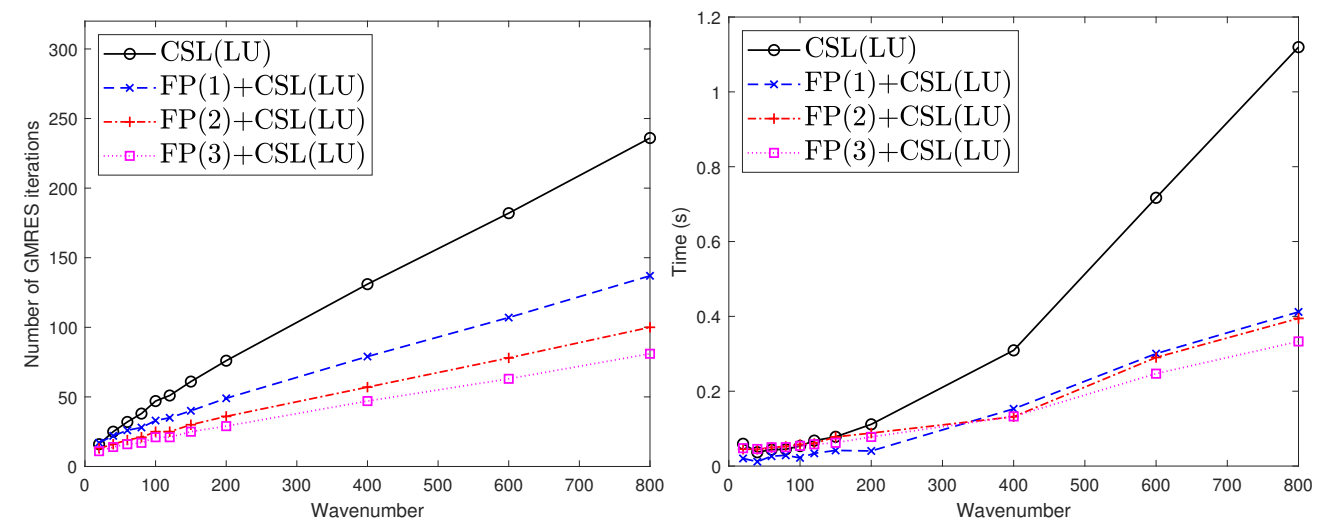

FIG. 4.1. Model problem 1: Number of GMRES iterations (left) and time elapsed (right) for computing the shifted Laplacian with an LU factorization.

where $f$ is a point source centered at $x=1 / 2$. We discretize this problem with second-order central differences on a uniform grid with 15 points per wavelength. This simple problem can be solved with a direct method, but we present it for reference.

First, we apply the Faber preconditioner of degree 1, 2, and 3 to accelerate full GMRES. The GMRES iteration is stopped when the relative residual is below $10^{-8}$. Table 4.1 and Figure 4.1 provide the number of GMRES iterations, the number of matrix-vector products (MV), and the time elapsed (in seconds) when the shifted Laplacian is computed using forward and backward substitution from a precomputed LU factorization (only for reference, since this is not done for practical problems). The timings include the computation of the LU factorization of $A_{\varepsilon}$. For comparison, we repeat the same experiment with the shifted Laplace preconditioner computed approximately with a multigrid $V(1,1)$-cycle and a Jacobi smoother with relaxation parameter $\omega=2 / 3$ and display the results in Table 4.2 and Figure 4.2.

In both experiments, we see a reduction in the number of iterations when the CSL is augmented with the Faber preconditioner. This reduction is larger for the Faber preconditioner of higher degrees when the LU factorization is used but not when the shifted Laplacian is approximately computed with a multigrid cycle, for which there is not a significant difference in the number of iterations for the Faber preconditioner of degrees 1, 2, and 3. 


\section{ETNA}

Kent State University and

Johann Radon Institute (RICAM)

TABLE 4.2

Model problem 1: Number of GMRES iterations, matrix-vector products, and timings for computing the shifted Laplacian with a multigrid cycle.

\begin{tabular}{|c|c|c|c|c|c|c|c|c|c|c|c|c|}
\hline \multirow[t]{2}{*}{$k$} & \multicolumn{3}{|c|}{ CSL(MG) } & \multicolumn{3}{|c|}{$\mathrm{FP}(1)+\mathrm{CSL}(\mathrm{MG})$} & \multicolumn{3}{|c|}{$\mathrm{FP}(2)+\mathrm{CSL}(\mathrm{MG})$} & \multicolumn{3}{|c|}{$\mathrm{FP}(3)+\mathrm{CSL}(\mathrm{MG})$} \\
\hline & Iter. & MV & Time(s) & Iter. & MV & Time(s) & Iter. & MV & Time(s) & Iter. & MV & Time(s) \\
\hline 20 & 17 & 17 & 0.059 & 16 & 32 & 0.099 & 13 & 39 & 0.096 & 12 & 48 & 0.103 \\
\hline 40 & 27 & 27 & 0.047 & 22 & 44 & 0.101 & 19 & 57 & 0.109 & 16 & 64 & 0.124 \\
\hline 60 & 36 & 36 & 0.065 & 30 & 60 & 0.150 & 27 & 81 & 0.148 & 22 & 88 & 0.147 \\
\hline 80 & 42 & 42 & 0.077 & 31 & 62 & 0.132 & 27 & 81 & 0.146 & 24 & 96 & 0.154 \\
\hline 100 & 52 & 52 & 0.110 & 51 & 102 & 0.194 & 44 & 132 & 0.196 & 41 & 164 & 0.210 \\
\hline 120 & 61 & 61 & 0.148 & 43 & 86 & 0.177 & 42 & 126 & 0.224 & 34 & 136 & 0.235 \\
\hline 150 & 71 & 71 & 0.166 & 49 & 98 & 0.214 & 40 & 120 & 0.200 & 37 & 148 & 0.246 \\
\hline 200 & 90 & 90 & 0.205 & 75 & 150 & 0.305 & 65 & 195 & 0.352 & 64 & 256 & 0.410 \\
\hline 400 & 163 & 163 & 0.530 & 120 & 240 & 0.680 & 107 & 321 & 0.792 & 109 & 436 & 0.948 \\
\hline 600 & 238 & 238 & 1.379 & 151 & 302 & 1.159 & 115 & 345 & 1.085 & 100 & 400 & 1.145 \\
\hline 800 & 305 & 305 & 2.069 & 210 & 420 & 1.679 & 186 & 558 & 1.765 & 198 & 792 & 2.144 \\
\hline
\end{tabular}
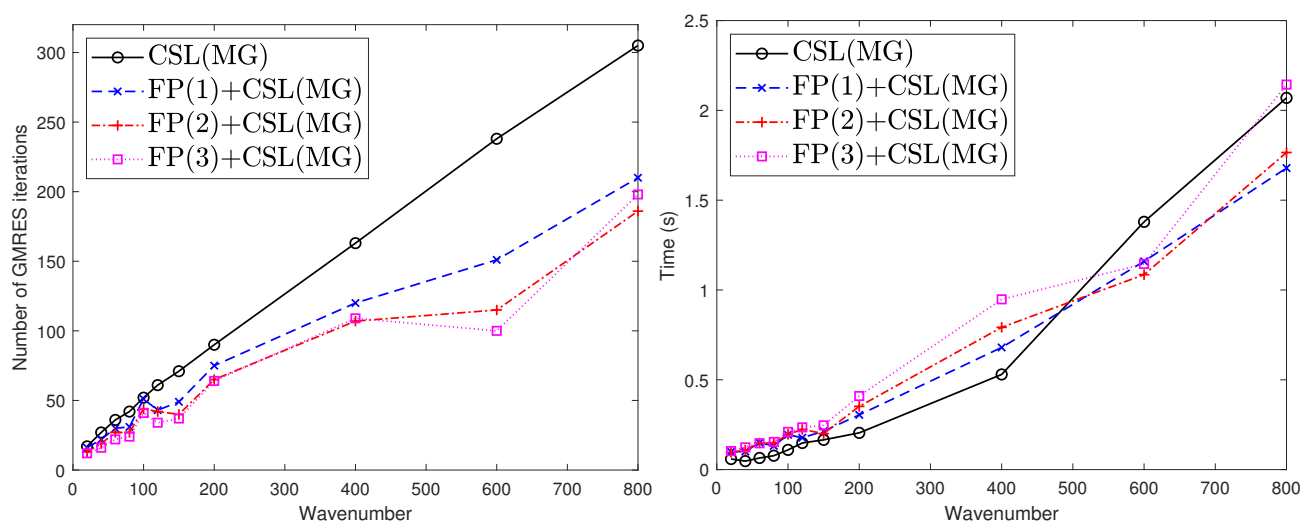

FIG. 4.2. Model problem 1: Number of GMRES iterations (left) and time elapsed (right) for computing the shifted Laplacian with a multigrid cycle.

When full GMRES is used, one cannot expect that the number of matrix-vector products is reduced, nevertheless we observe some speedup when the LU factorization is used since the extra cost of applying the preconditioner per iteration is negligible and the number of operations for orthogonalization is reduced. When the preconditioner is approximately computed via the multigrid method, the application of the preconditioner is a more expensive operation, and the reduction in the number of iterations is not as large as when the CSL is computed with backward and forward substitution. Therefore there is no speedup.

4.2. Model problem 2. We consider a Helmholtz problem in one dimension with absorbing boundary conditions:

$$
\begin{aligned}
-u^{\prime \prime}-k^{2} u & =f \quad \text { in }(0,1), \\
u^{\prime}(0)+i k u(0) & =0, \\
u^{\prime}(1)-i k u(1) & =0,
\end{aligned}
$$

where $f$ is a point source centered at $x=1 / 2$. We discretize this problem on a uniform grid with piecewise linear finite elements and 15 points per wavelength.

As in model problem 1, we apply the Faber preconditioner to accelerate full GMRES. The results are very similar to the previous experiment. We observe a decrease in the number 


\section{ETNA}

Kent State University and

Johann Radon Institute (RICAM)

TABLE 4.3

Model problem 2: Number of GMRES iterations, matrix-vector products, and timings for computing the shifted Laplacian with an LU factorization.

\begin{tabular}{|c|c|c|c|c|c|c|c|c|c|c|c|c|}
\hline \multirow[t]{2}{*}{$k$} & \multicolumn{3}{|c|}{ CSL(LU) } & \multicolumn{3}{|c|}{$\mathrm{FP}(1)+\mathrm{CSL}(\mathrm{LU})$} & \multicolumn{3}{|c|}{$\mathrm{FP}(2)+\mathrm{CSL}(\mathrm{LU})$} & \multicolumn{3}{|c|}{$\mathrm{FP}(3)+\mathrm{CSL}(\mathrm{LU})$} \\
\hline & Iter. & MV & Time(s) & Iter. & MV & Time(s) & Iter. & MV & Time(s) & Iter. & MV & Time(s) \\
\hline 20 & 18 & 18 & 0.036 & 19 & 38 & 0.011 & 16 & 48 & 0.044 & 13 & 52 & 0.049 \\
\hline 40 & 28 & 28 & 0.041 & 25 & 50 & 0.014 & 19 & 57 & 0.047 & 16 & 64 & 0.048 \\
\hline 60 & 36 & 36 & 0.045 & 29 & 58 & 0.028 & 22 & 66 & 0.054 & 18 & 72 & 0.053 \\
\hline 80 & 42 & 42 & 0.065 & 31 & 62 & 0.031 & 23 & 69 & 0.053 & 20 & 80 & 0.054 \\
\hline 100 & 50 & 50 & 0.057 & 36 & 72 & 0.030 & 27 & 81 & 0.068 & 23 & 92 & 0.056 \\
\hline 120 & 55 & 55 & 0.072 & 38 & 76 & 0.036 & 28 & 84 & 0.082 & 24 & 96 & 0.063 \\
\hline 150 & 65 & 65 & 0.087 & 43 & 86 & 0.044 & 32 & 96 & 0.076 & 27 & 108 & 0.066 \\
\hline 200 & 80 & 80 & 0.111 & 51 & 102 & 0.041 & 38 & 114 & 0.086 & 31 & 124 & 0.087 \\
\hline 400 & 135 & 135 & 0.313 & 81 & 162 & 0.153 & 59 & 177 & 0.144 & 48 & 192 & 0.137 \\
\hline 600 & 188 & 188 & 1.051 & 111 & 222 & 0.439 & 80 & 240 & 0.366 & 65 & 260 & 0.312 \\
\hline 800 & 241 & 241 & 1.624 & 140 & 280 & 0.624 & 102 & 306 & 0.505 & 83 & 332 & 0.419 \\
\hline
\end{tabular}
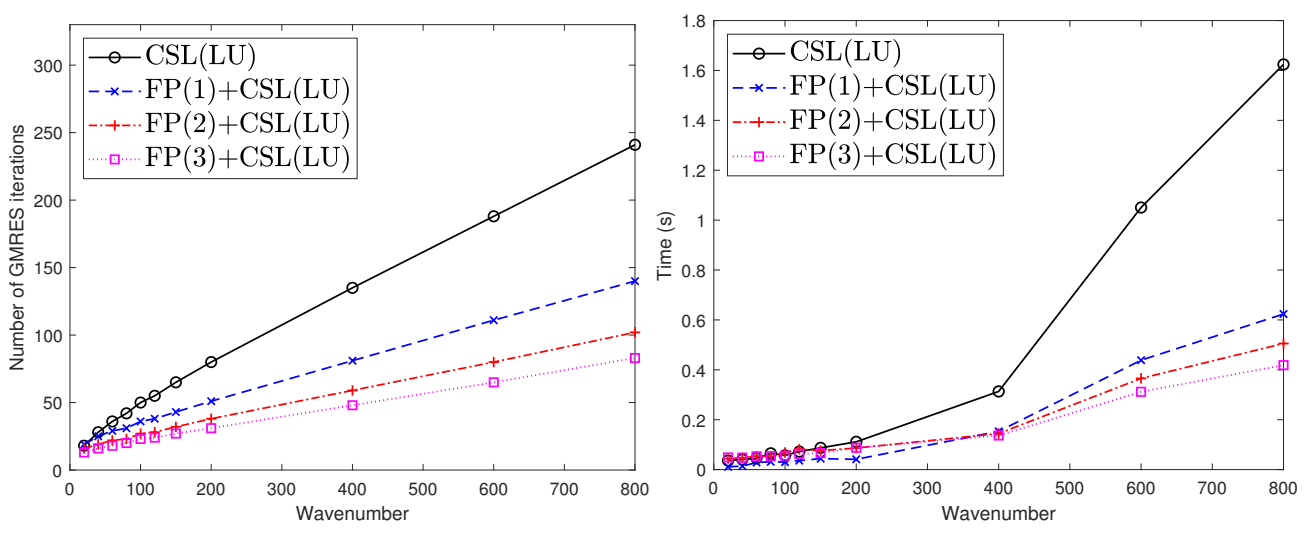

FIG. 4.3. Model problem 2: Number of GMRES iterations (left) and time elapsed (right) for computing the shifted Laplacian with an LU factorization.

of iterations which results in a speedup (the computation time is typically halved for degree $n=1$, while the computation time is reduced by more than a half for higher wavenumbers for degrees 2 and 3) when the preconditioner is inverted using forward and backward substitution with a precomputed LU decomposition; see Table 4.3 and Figure 4.3. When a multigrid cycle is used, the decrease in the number of iterations is not as significant, and it does not translate to a faster iteration; see Table 4.4 and Figure 4.4.

4.3. Model problem 3. We consider a Helmholtz problem in two dimensions with absorbing boundary conditions:

$$
\begin{aligned}
-\Delta u-k^{2} u=f & \text { in } \Omega=(0,1)^{2}, \\
\partial_{n} u-i k u=0 & \text { in } \partial \Omega,
\end{aligned}
$$

where $f$ is a point source centered at $(1 / 2,1 / 2)$. This problem is discretized with second-order finite differences on a uniform grid with 12 points per wavelength [46].

We solve this problem with restarted and full GMRES. We begin with restarted GMRES and set the length of an outer cycle equal to 20 (i.e., we use GMRES(20)), and the wavenumber is $k=50$. Here we set the degree of the Faber preconditioner to be equal to 1,2 , or 3 and plot the norm of the residual as a function of the number of matrix-vector products and use an 
TABLE 4.4

Model problem 2: Number of GMRES iterations, matrix-vector products, and timings for computing the shifted Laplacian with a multigrid cycle.

\begin{tabular}{ccccccccccccc}
$k$ & \multicolumn{4}{c}{ CSL(MG) } & \multicolumn{3}{c}{ FP(1)+CSL(MG) } & \multicolumn{3}{c}{ FP(2)+CSL(MG) } & \multicolumn{3}{c}{ FP(3)+CSL(MG) } \\
& Iter. & MV & Time(s) & Iter. & MV & Time(s) & Iter. & MV & Time(s) & Iter. & MV & Time(s) \\
\hline 20 & 20 & 20 & 0.051 & 17 & 34 & 0.122 & 15 & 45 & 0.101 & 13 & 52 & 0.111 \\
40 & 29 & 29 & 0.051 & 24 & 48 & 0.134 & 20 & 60 & 0.117 & 17 & 68 & 0.121 \\
60 & 40 & 40 & 0.072 & 32 & 64 & 0.147 & 28 & 84 & 0.146 & 24 & 96 & 0.167 \\
80 & 45 & 45 & 0.087 & 33 & 66 & 0.173 & 28 & 84 & 0.162 & 25 & 100 & 0.168 \\
100 & 56 & 56 & 0.112 & 52 & 104 & 0.191 & 44 & 132 & 0.198 & 43 & 172 & 0.232 \\
120 & 65 & 65 & 0.149 & 46 & 92 & 0.188 & 44 & 132 & 0.227 & 36 & 144 & 0.251 \\
150 & 74 & 74 & 0.171 & 51 & 102 & 0.210 & 42 & 126 & 0.223 & 38 & 152 & 0.251 \\
200 & 93 & 93 & 0.206 & 77 & 154 & 0.322 & 65 & 195 & 0.355 & 66 & 264 & 0.455 \\
400 & 167 & 167 & 0.557 & 123 & 246 & 0.692 & 107 & 321 & 0.796 & 110 & 440 & 0.976 \\
600 & 242 & 242 & 1.959 & 153 & 306 & 1.392 & 117 & 351 & 1.244 & 102 & 408 & 1.258 \\
800 & 310 & 310 & 2.992 & 213 & 426 & 2.143 & 188 & 564 & 2.130 & 199 & 796 & 2.586
\end{tabular}
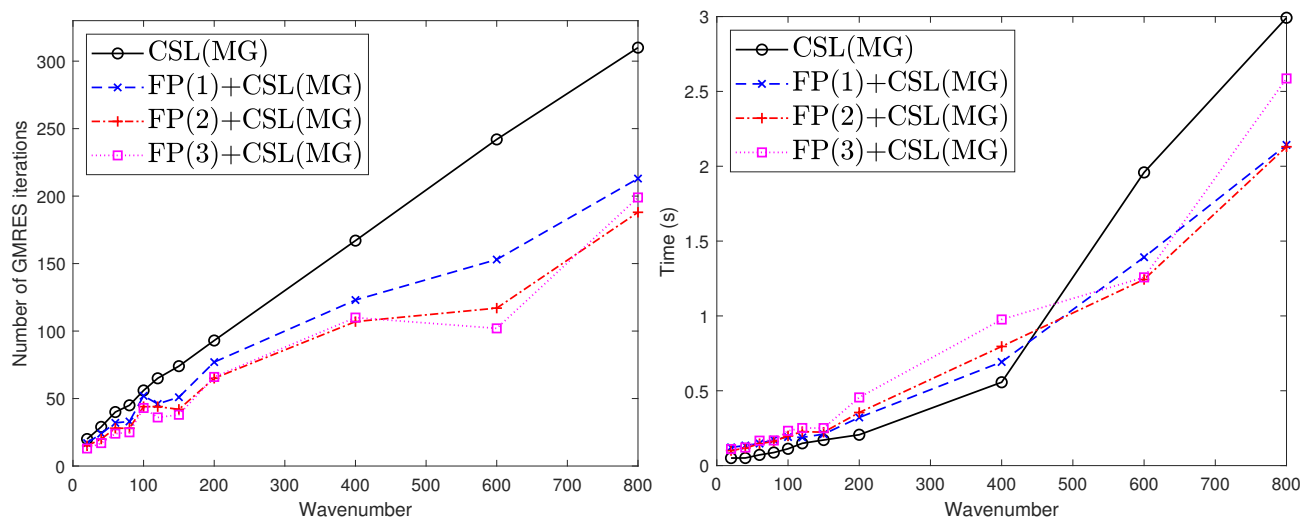

FIG. 4.4. Model problem 2: Number of GMRES iterations (left) and time elapsed (right) for computing the shifted Laplacian with a multigrid cycle.

$F(1,1)$-cycle for the multigrid solve of the shifted Laplace preconditioner and shift parameter $\varepsilon=0.5 k^{2}$. The iteration is stopped when the residual is reduced by a factor of $10^{-8}$. The results are shown in Figure 4.5 (top left). The Faber preconditioner does not reduce the number of matrix-vector products, but since there is only a slight increase in matrix-vector products, there may be a potential speedup from the reduction in the number of vector operations in GMRES. This will be explored in the subsequent experiments. We also see that there is no significant gain in using the Faber preconditioner of degree higher than 1 . Repeating the experiment with full GMRES instead of GMRES(20) yields very similar results; see Figure 4.5 (top right). For $k=100$, the results are similar (see Figure 4.5 (bottom)), and they show that no significant gain is obtained from a higher-degree truncated Faber series with only a slight increase in the number of matrix-vector products compared to the standalone CSL preconditioner.

Next, we solve model problem 3 for different wavenumbers. First, we use GMRES(20) and compare the standalone CSL preconditioner and the CSL combined with the Faber preconditioner of degrees 1 and 2. The number of GMRES(20) iterations and timings for increasing wavenumbers are presented in Figure 4.6 (top) and in Table 4.5. We can see that, with respect to the standalone CSL preconditioner, there is a decrease of $40 \%$ to $45 \%$ in the number of iterations ((iter. CSL - iter. CSL+FP $(n))$ /iter. CSL) when applying the Faber 


\section{ETNA}

Kent State University and

Johann Radon Institute (RICAM)
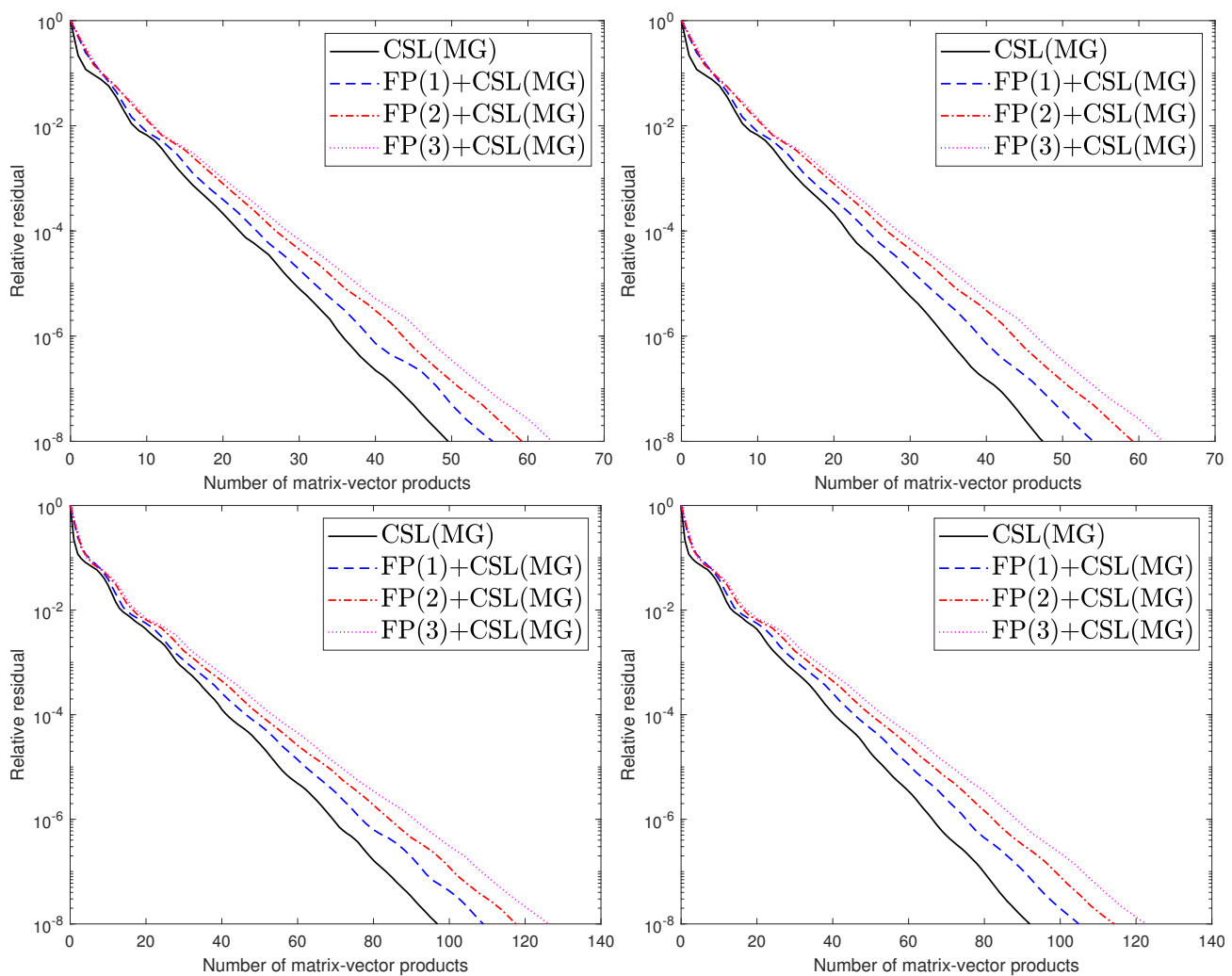

FIG. 4.5. Model problem 3 with $k=50$ (top) and $k=100$ (bottom): Relative residual as a function of the number of matrix-vector products when preconditioning by the CSL and the Faber preconditioner with varying degrees. Left: GMRES(20). Right: full GMRES.

preconditioner of degree 1. With the Faber preconditioner of degree 2, the number of iterations is further reduced. However, there is no speedup, which is due to the fact that the number of matrix-vector products (multigrid solves of the CSL preconditioner) is not reduced. We repeat this experiment with full GMRES and display the results in Figure 4.6 (bottom) and Table 4.6. Similarly as before, applying the Faber preconditioner of degree 1 leads to a reduction in the number of iterations by $40 \%$ to $45 \%$ in comparison with the standalone CSL, and this translates to a speedup of approximately $33 \%$ to $35 \%$ for the larger wavenumbers.

4.4. Model problem 4. We now consider a Helmholtz problem in two dimensions with variable wavenumber and absorbing boundary conditions, similar to the ones proposed in $[20,38]$ :

$$
\begin{aligned}
-\Delta u-k^{2} u=f & \text { in } \Omega=(0,1)^{2}, \\
\partial_{n} u-i k u=0 & \text { in } \partial \Omega,
\end{aligned}
$$

where $f$ is a point source centered at $(1 / 2,1 / 2)$. The space-dependent wavenumber is

$$
k(x, y)=\left\{\begin{array}{llr}
(4 / 3) k_{\mathrm{ref}} & \text { if } & 0 \leq y<0.2 x+0.2 \\
k_{\mathrm{ref}} & \text { if } & 0.2 x+0.2 \leq y<-0.2 x+0.8 \\
2 k_{\mathrm{ref}} & \text { if } & -0.2 x+0.8 \leq y<1
\end{array}\right.
$$




\section{ETNA}

Kent State University and Johann Radon Institute (RICAM)
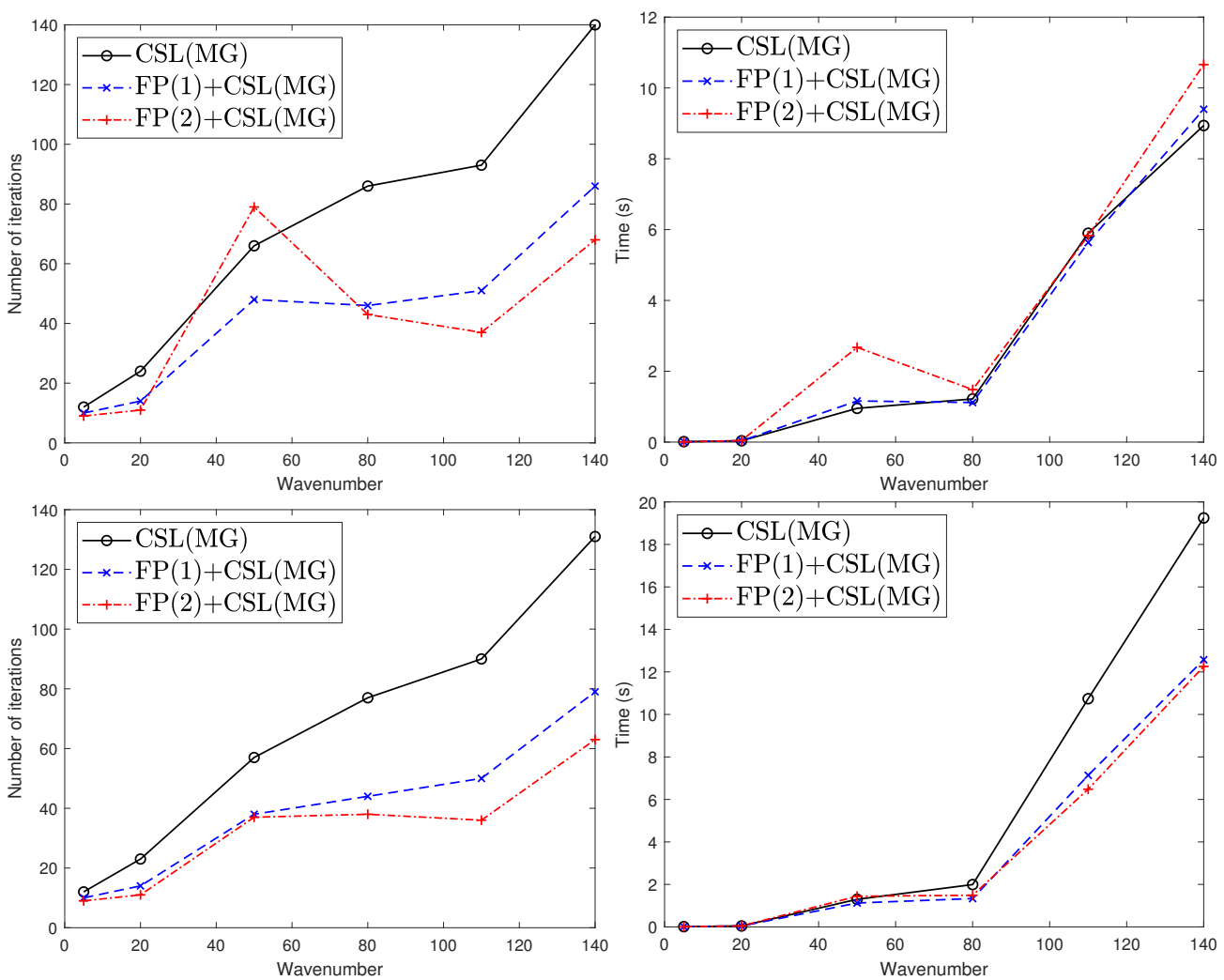

FIG. 4.6. Model problem 3 with GMRES(20) (top) and full GMRES (bottom): Number of iterations (left) and time in seconds (right) as a function of the wavenumber when preconditioning with the standalone CSL and the CSL combined with the Faber preconditioner with degrees 1 and 2.

where $k_{\text {ref }} \in \mathbb{R}$. This problem is discretized with second-order finite differences on a uniform grid with 12 points per wavelength [46].

Since restarted GMRES performs very poorly for this problem, we only use full GMRES and compare the standalone CSL preconditioner with the CSL combined with the Faber preconditioner of degrees 1 and 2. The number of GMRES iterations and timings for increasing wavenumbers are given in Figure 4.7 and in Table 4.7. As in the case of a constant wavenumber, we see that compared to the CSL preconditioner, applying the Faber preconditioner of degree 1 leads to a decrease in the number of iterations of approximately $40 \%$ and a reduction in CPU time of around $20 \%$ (and up to $40 \%$ for certain $k$ ).

5. Conclusions. In this paper we have introduced the Faber preconditioner for the discretized Helmholtz equation preconditioned with the complex shifted Laplacian. The Faber preconditioner is a polynomial preconditioner constructed by truncating the Faber series of $1 / z$ on a non-convex 'bratwurst' set, which contains the eigenvalues of the CSL-preconditioned Helmholtz matrix. The Faber polynomials can be computed efficiently with a three-term recurrence, and we give analytic expressions for the coefficients of the Faber series. Finally, we show that the low-order Faber preconditioners are indeed nonsingular operators.

Our numerical experiments in $1 \mathrm{D}$ and $2 \mathrm{D}$ with constant and varying wavenumbers show that the Faber preconditioner typically reduces the number of GMRES iterations. When using 


\section{ETNA}

Kent State University and

Johann Radon Institute (RICAM)

THE FABER PRECONDITIONER FOR THE HELMHOLTZ EQUATION

TABLE 4.5

Model problem 3 with GMRES(20): Number of GMRES iterations, matrix-vector products, and timings for computing the shifted Laplacian with a multigrid cycle.

\begin{tabular}{cccccccccc}
$k$ & \multicolumn{3}{c}{ CSL(MG) } & \multicolumn{3}{c}{ FP(1)+CSL(MG) } & \multicolumn{2}{c}{ FP(2)+CSL(MG) } \\
& Iter. & MV & Time(s) & Iter. & MV & Time(s) & Iter. & MV & Time(s) \\
\hline 5 & 12 & 12 & 0.012 & 10 & 20 & 0.010 & 9 & 27 & 0.007 \\
20 & 24 & 24 & 0.037 & 14 & 28 & 0.037 & 11 & 33 & 0.043 \\
50 & 66 & 66 & 0.953 & 48 & 96 & 1.161 & 79 & 237 & 2.674 \\
80 & 86 & 86 & 1.219 & 46 & 92 & 1.117 & 43 & 129 & 1.482 \\
110 & 93 & 93 & 5.898 & 51 & 102 & 5.635 & 37 & 111 & 5.828 \\
140 & 140 & 140 & 8.939 & 86 & 172 & 9.398 & 68 & 204 & 10.660
\end{tabular}

TABLE 4.6

Model problem 3 with full GMRES: Number of GMRES iterations, matrix-vector products, and timings for computing the shifted Laplacian with a multigrid cycle.

\begin{tabular}{cccccccccc}
$k$ & \multicolumn{3}{c}{ CSL(MG) } & \multicolumn{3}{c}{ FP(1)+CSL(MG) } & \multicolumn{3}{c}{ FP(2)+CSL(MG) } \\
& Iter. & MV & Time(s) & Iter. & MV & Time(s) & Iter. & MV & Time(s) \\
\hline 5 & 12 & 12 & 0.013 & 10 & 20 & 0.011 & 9 & 27 & 0.007 \\
20 & 23 & 23 & 0.044 & 14 & 28 & 0.045 & 11 & 33 & 0.046 \\
50 & 57 & 57 & 1.298 & 38 & 76 & 1.125 & 37 & 111 & 1.443 \\
80 & 77 & 77 & 1.992 & 44 & 88 & 1.335 & 38 & 114 & 1.490 \\
110 & 90 & 90 & 10.739 & 50 & 100 & 7.135 & 36 & 108 & 6.481 \\
140 & 131 & 131 & 19.247 & 79 & 158 & 12.574 & 63 & 189 & 12.256
\end{tabular}

full GMRES, we usually observe a reduction in the number of iterations and in CPU time, in particular for higher wavenumbers, which is due to the reduced number of vector operations.

As an outlook, the Faber preconditioner could be combined with deflation techniques. It is known [25] that the eigenvalues of the deflated CSL-preconditioned Helmholtz operator are shifted away from the origin and still lie in the disk $\{z \in \mathbb{C}:|z-1 / 2| \leq 1 / 2\}$, ensuring that the Faber preconditioner can be applied.

\section{Appendix A. Boundary rotation.}

In this section we discuss the boundary rotation of our inclusion set. We begin with the definition of the total rotation of a curve. The total rotation of a smooth Jordan curve $\Gamma$, parametrised by $\gamma:[a, b] \rightarrow \mathbb{C}$ with $\gamma^{\prime}(t) \neq 0$, is

$$
V(\Gamma)=\int_{a}^{b}\left|\frac{d}{d t} \arg \left(\gamma^{\prime}(t)\right)\right| d t
$$

where $[a, b] \ni t \mapsto \arg \left(\gamma^{\prime}(t)\right)$ denotes a smooth branch of the argument. Geometrically, the total rotation is the total variation of the angle $\theta$ between the tangent to $\Gamma$ and the positive real line and can also be written in the form $V=\int_{\Gamma}|d \theta|$. This definition can be extended to Jordan arcs by integrating along each "side" and "around the corners" (see [14]) and to arbitrary Jordan curves by a limiting process; see [32, 44]. For a compact set $E$ bounded by a Jordan curve, we define $V=V(E)=V(\partial E)$, and $E$ has bounded boundary rotation if $V<\infty$; see [14, 31,32, 44]. We have $V \geq 2 \pi$, and $V=2 \pi$ if and only if $E$ is convex.

The set $\Omega=\Omega(\lambda, \phi, \sigma)$ in Theorem 3.2 has bounded boundary rotation; see [31, Lemma 3.5]. Then $E=\frac{1}{2} \Omega+\frac{1}{2}$ has the same boundary rotation, $V(E)=V(\Omega)$, which is easily seen from (A.1). Let us discuss $V(\Omega)$. 

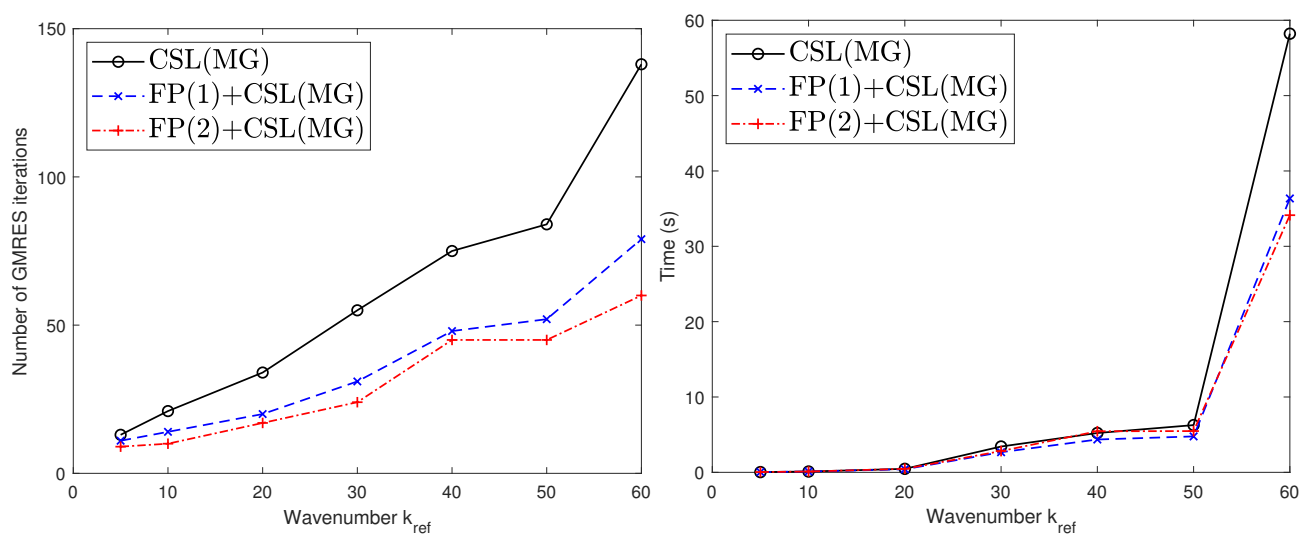

FIG. 4.7. Model problem 4: Number of GMRES iterations (left) and time in seconds (right) as a function of the

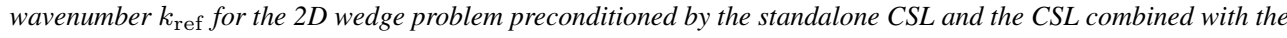
Faber preconditioner with degrees 1 and 2 .

TABLE 4.7

Model problem 4: Number of GMRES iterations, matrix-vector products, and timings for computing the shifted Laplacian with a MG cycle.

\begin{tabular}{cccccccccc}
$k_{\text {ref }}$ & \multicolumn{3}{c}{ CSL(MG) } & \multicolumn{3}{c}{$\mathrm{FP}(1)+\mathrm{CSL}(\mathrm{MG})$} & \multicolumn{2}{c}{$\mathrm{FP}(2)+\mathrm{CSL}(\mathrm{MG})$} \\
& Iter. & MV & Time(s) & Iter. & MV & Time(s) & Iter. & MV & Time(s) \\
\hline 5 & 13 & 13 & 0.031 & 11 & 22 & 0.036 & 9 & 27 & 0.040 \\
10 & 21 & 21 & 0.106 & 14 & 28 & 0.108 & 10 & 30 & 0.107 \\
20 & 34 & 34 & 0.464 & 20 & 40 & 0.410 & 17 & 51 & 0.467 \\
30 & 55 & 55 & 3.426 & 31 & 62 & 2.673 & 24 & 72 & 2.823 \\
40 & 75 & 75 & 5.219 & 48 & 96 & 4.357 & 45 & 135 & 5.452 \\
50 & 84 & 84 & 6.269 & 52 & 104 & 4.767 & 45 & 135 & 5.476 \\
60 & 138 & 138 & 58.220 & 79 & 158 & 36.349 & 60 & 180 & 34.130
\end{tabular}

For $\sigma=1$, the set $\Omega=\Omega(\lambda, \phi, 1)$ is the circular $\operatorname{arc}\left\{\lambda e^{i \beta}: \frac{\phi}{2} \leq \beta \leq 2 \pi-\frac{\phi}{2}\right\}$ (see [31, Example 3.2]), which has interior angle $2 \pi-\phi$. Its boundary rotation then is

$$
V=6 \pi-2 \phi
$$

See also [6, p. 200] for the boundary rotation of an annular sector.

For $1<\sigma<P$, the set $\Omega$ has an analytic boundary which can be parametrized by $\gamma(t)=\psi\left(e^{i t}\right), 0 \leq t \leq 2 \pi$. Then,

$$
\frac{d}{d t} \arg \left(\gamma^{\prime}(t)\right)=1+\frac{d}{d t} \arg \left(\psi^{\prime}\left(e^{i t}\right)\right)=1+\operatorname{Re}\left(\frac{e^{i t} \psi^{\prime \prime}\left(e^{i t}\right)}{\psi^{\prime}\left(e^{i t}\right)}\right) .
$$

The last equality is well known and can be established as follows:

$$
\begin{aligned}
\frac{d}{d t} \arg \left(\psi^{\prime}\left(e^{i t}\right)\right) & =\frac{d}{d t} \operatorname{Re}\left(-i \log \left|\psi^{\prime}\left(e^{i t}\right)\right|+\arg \left(\psi^{\prime}\left(e^{i t}\right)\right)\right)=\frac{d}{d t} \operatorname{Re}\left(-i \log \left(\psi^{\prime}\left(e^{i t}\right)\right)\right) \\
& =\operatorname{Re}\left(\frac{e^{i t} \psi^{\prime \prime}\left(e^{i t}\right)}{\psi^{\prime}\left(e^{i t}\right)}\right) .
\end{aligned}
$$

Hence, the boundary rotation of $\Omega$ is

$$
V=\int_{0}^{2 \pi}\left|1+\operatorname{Re}\left(\frac{e^{i t} \psi^{\prime \prime}\left(e^{i t}\right)}{\psi^{\prime}\left(e^{i t}\right)}\right)\right| d t
$$




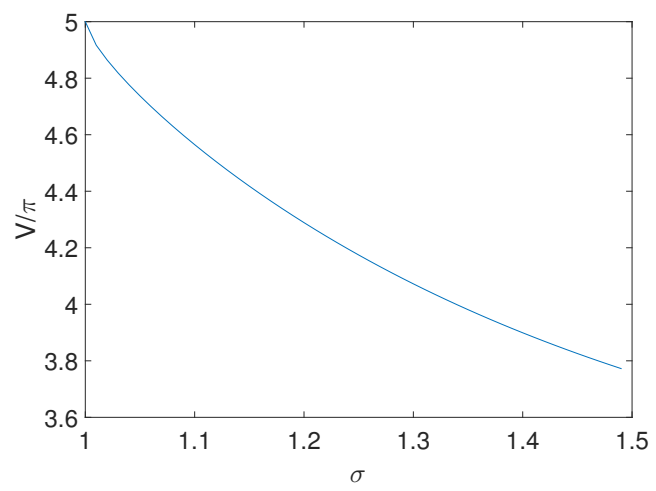

FIG. A.1. Boundary rotation $V / \pi$ for $\Omega(-1, \pi / 2, \sigma)$ as a function of $\sigma$.

Using the map $\psi$ from Theorem 3.2, we find

$$
\frac{w \psi^{\prime \prime}(w)}{\psi^{\prime}(w)}=\frac{2 w^{2}+2 w S}{w^{2}+w 2 S-\lambda^{2} M N-\lambda(M+N) S}-\frac{2 w}{w+S}
$$

where $S=\lambda \frac{N M-1}{N-M}$. Together with (A.2), it is easy to compute the boundary rotation $V$ numerically. Figure A.1 displays the computed values $V / \pi$ for the sets $\Omega(-1, \pi / 2, \sigma)$ with $1<\sigma<P=1.4966$ (rounded to four digits); see Figure 3.2 for the shape of $\Omega$.

\section{Appendix B. Proof of Proposition 3.4.}

Proof of Proposition 3.4. Recall the expressions (3.7) for the Faber polynomials and (3.9) for the coefficients. The truncated Faber series of degree $n=1$ is

$$
s_{1}(z)=a_{1} F_{1}(z)+a_{0} F_{0}(z)=-\frac{\rho-M}{\rho^{2}} F_{1}(z)+\frac{\rho-M}{\rho}=-\frac{\rho-M}{\rho^{2}}\left(F_{1}(z)-\rho\right) .
$$

Thus, the unique zero $z_{1}$ of $s_{1}$ is the zero of

$$
F_{1}(z)-\rho=2(N-M) z-2 N-\frac{M N-1}{N-M}-\rho,
$$

i.e.,

$$
z_{1}=\frac{2 N+\rho+\frac{M N-1}{N-M}}{2(N-M)}
$$

We have $z_{1}>1$ since the numerator satisfies

$$
\begin{aligned}
2 N+\rho+\frac{M N-1}{N-M} & >3 N+\frac{M N-1}{N-M}=2 N+\frac{N^{2}-M N+M N-1}{N-M} \\
& >2 N \geq 2(N-M)>0 .
\end{aligned}
$$

Thus, the zero of $s_{1}$ is not in the spectrum of $A A_{\varepsilon}^{-1}$ and $s_{1}\left(A A_{\varepsilon}^{-1}\right)$ is nonsingular.

For $n=2$, the truncated Faber series is

$$
s_{2}(z)=a_{2} F_{2}(z)+a_{1} F_{1}(z)+a_{0} F_{0}(z)=\frac{\rho-M}{\rho^{3}}\left(F_{2}(z)-\rho F_{1}(z)+\rho^{2}\right) .
$$


Therefore, the zeros of $s_{2}$ are the zeros of $p(z)=F_{2}(z)-\rho F_{1}(z)+\rho^{2}$. We have

$$
p(z)=a z^{2}+b z+c,
$$

where the coefficients are given by

$$
\begin{aligned}
& a=4(N-M)^{2}, \\
& b=-8 N^{2}+4 M N+4-2 \rho(N-M), \\
& c=4 N^{2}-2-S^{2}+2 N \rho+\rho S+\rho^{2},
\end{aligned}
$$

and where $S=(M N-1) /(N-M)$. Note that all coefficients are real.

We first show that $p$ has no real roots and equivalently that the discriminant of $p$ is negative. Let $\Delta=(b / 2)^{2}-a c$ be (one fourth) of the discriminant. Then

$$
\Delta=\left(2-4 N^{2}+2 M N-\rho(N-M)\right)^{2}-4(N-M)^{2}\left(4 N^{2}-2-S^{2}+2 N \rho+\rho S+\rho^{2}\right) .
$$

Simplifying this expression (all computations in this paragraph have been performed with Mathematica 11.0) yields

$$
\begin{aligned}
\Delta=8 & +11 M^{2}-22 M N-5 N^{2}-14 M^{2} N^{2}+28 M N^{3}-6 N^{4} \\
& -6 N(M-N)^{2} \sqrt{N^{2}-1} .
\end{aligned}
$$

The term with the square root is negative, so it is sufficient to show that the remaining term is also negative. The properties $0 \leq M<1<N$ are not sufficient to prove that this first term is negative, so we work with the expressions of $M$ and $N$ in $\phi$ and $\sigma$ from Theorem 3.2. Inserting these yields a positive factor times the term

$$
\begin{aligned}
& -\left(1-8 \sigma^{2}+\sigma^{4}\right) \cos (\phi / 2)+\left(1+\sigma^{2}\right)^{2} \cos (\phi) \\
& \quad+2\left(-7+3 \sigma^{2}-7 \sigma^{4}+8\left(\sigma^{4}-1\right) \sin (\phi / 4)+2\left(\sigma^{4}-1\right) \sin (3 \phi / 4)\right) .
\end{aligned}
$$

It is now sufficient to show that this term is negative for all $0<\phi<2 \pi$ and admissible $\sigma$. For fixed $0<\phi<2 \pi$, we substitute $\tau=\sigma^{2}$ to obtain a quadratic polynomial in $\tau$ :

$$
\begin{aligned}
q(\tau)=(-1+8 \tau & \left.-\tau^{2}\right) \cos (\phi / 2)+(1+\tau)^{2} \cos (\phi) \\
& -14+6 \tau-14 \tau^{2}+16\left(\tau^{2}-1\right) \sin (\phi / 4)+4\left(\tau^{2}-1\right) \sin (3 \phi / 4) .
\end{aligned}
$$

The derivative of $q$ is

$$
q^{\prime}(\tau)=(8-2 \tau) \cos (\phi / 2)+2(1+\tau) \cos (\phi)+6-28 \tau+32 \tau \sin (\phi / 4)+8 \tau \sin (3 \phi / 4),
$$

which has the unique zero

$$
\tau_{0}=\frac{4(\cos (\phi / 8)+\sin (\phi / 8))^{4}}{5+2 \cos (\phi / 2)}
$$

Then

$$
q\left(\tau_{0}\right)=-\frac{6(7+4 \cos (\phi / 2))(\cos (\phi / 8)+\sin (\phi / 8))^{4}}{5+2 \cos (\phi / 2)}<0
$$

for all $0<\phi<2 \pi$. The function $q$ has a (global) maximum at this point since the coefficient of $\tau^{2}$ in $q$ is

$$
\begin{aligned}
-\cos (\phi / 2)+\cos (\phi) & -14+16 \sin (\phi / 4)+4 \sin (3 \phi / 4) \\
& =-2(5+2 \cos (\phi / 2))(\cos (\phi / 8)-\sin (\phi / 8))^{4}<0
\end{aligned}
$$




\section{ETNA}

Kent State University and

Johann Radon Institute (RICAM)

for all $0<\phi<2 \pi$. This shows that $q(\tau)<0$ for all $\tau$ and $\phi$, which implies that $\Delta<0$ and thus that $p$ has no real roots.

Hence the polynomial $p$ has a pair of conjugate roots $\lambda, \bar{\lambda}$ and can be written in the form

$$
p(z)=a\left(z^{2}+\frac{b}{a} z+\frac{c}{a}\right)=a(z-\lambda)(z-\bar{\lambda})=a\left(z^{2}-2 \operatorname{Re}(\lambda) z+|\lambda|^{2}\right) .
$$

This gives the relations

$$
\lambda \bar{\lambda}=|\lambda|^{2}=\frac{c}{a}, \quad \operatorname{Re}(\lambda)=-\frac{b}{2 a} .
$$

We show now that the roots satisfy $|\lambda-1 / 2|>1 / 2$. Since

$$
\left|\lambda-\frac{1}{2}\right|^{2}=\left(\operatorname{Re}(\lambda)-\frac{1}{2}\right)^{2}+\operatorname{Im}(\lambda)^{2}=|\lambda|^{2}-\operatorname{Re}(\lambda)+\frac{1}{4}=\frac{c+\frac{b}{2}}{a}+\frac{1}{4}
$$

and $a>0$, we have that $|\lambda-1 / 2|>1 / 2$ is equivalent to $c+\frac{b}{2}>0$. We compute

$$
\begin{aligned}
c+\frac{b}{2} & =-S^{2}+2 N \rho+\rho S+\rho^{2}+2 M N-\rho(N-M) \\
& =\left(\rho^{2}-S^{2}\right)+\rho(S+N+M)+2 M N .
\end{aligned}
$$

To prove that $c+b / 2>0$, we show next that $S+N+M$ and $\rho^{2}-S^{2}$ are positive. A short computation yields

$$
S+N+M=\frac{N^{2}-1+M(N-M)}{N-M}>0
$$

since $N>1>M \geq 0$. Moreover, we have

$$
\rho^{2}-S^{2}=2 N \sqrt{N^{2}-1}+\frac{\left(2 N^{2}-1\right)(N-M)^{2}-(M N-1)^{2}}{(N-M)^{2}} .
$$

Expanding and rearranging the numerator of the last term, we obtain

$$
\begin{aligned}
\left(2 N^{2}-1\right)\left(N^{2}-\right. & \left.2 M N+M^{2}\right)-\left(M^{2} N^{2}-2 M N+1\right) \\
& =\left(N^{2}-1\right)\left(2(N-M)^{2}+\left(1-M^{2}\right)\right)>0,
\end{aligned}
$$

since $0 \leq M<1<N$. Therefore $\rho^{2}-S^{2}>0$, and, with the previous calculation, this implies that $c+b / 2>0$. We conclude that the roots of $s_{2}$ are outside the disk $\left|z-\frac{1}{2}\right| \leq \frac{1}{2}$, so they are not eigenvalues of $A A_{\varepsilon}^{-1}$, and $s_{2}\left(A A_{\varepsilon}^{-1}\right)$ is nonsingular.

\section{REFERENCES}

[1] T. Airaksinen, E. Heik kola, A. Pennanen, and J. Toivanen, An algebraic multigrid based shiftedLaplacian preconditioner for the Helmholtz equation, J. Comput. Phys., 226 (2007), pp. 1196-1210.

[2] A. BAYliss, C. I. Goldstein, AND E. TURKEL, On accuracy conditions for the numerical computation of waves, J. Comput. Phys., 59 (1985), pp. 396-404.

[3] B. BECKERMANN AND L. REICHEL, Error estimates and evaluation of matrix functions via the Faber transform, SIAM J. Numer. Anal., 47 (2009), pp. 3849-3883.

[4] M. BollhöFer, M. J. Grote, AND O. SChEnk, Algebraic multilevel preconditioner for the Helmholtz equation in heterogeneous media, SIAM J. Sci. Comput., 31 (2009), pp. 3781-3805.

[5] P.-H. CocQuet AND M. J. GANDER, How large a shift is needed in the shifted Helmholtz preconditioner for its effective inversion by multigrid?, SIAM J. Sci. Comput., 39 (2017), pp. A438-A478. 
[6] J. P. Coleman And N. J. Myers, The Faber polynomials for annular sectors, Math. Comp., 64 (1995), pp. 181-203, S1-S6.

[7] S. COOLS AND W. VANROOSE, Local Fourier analysis of the complex shifted Laplacian preconditioner for Helmholtz problems, Numer. Linear Algebra Appl., 20 (2013), pp. 575-597.

[8] - On the optimality of shifted Laplacian in a class of polynomial preconditioners for the Helmholtz equation, in Modern Solvers for Helmholtz Problems, D. Lahaye, J. Tang, and K. Vuik, eds., Geosyst. Math., Birkhäuser/Springer, Cham, 2017, pp. 53-81.

[9] J. H. CURTISs, Faber polynomials and the Faber series, Amer. Math. Monthly, 78 (1971), pp. 577-596.

[10] P. F. Dubois, A. GReEnbaum, AND G. H. Rodrigue, Approximating the inverse of a matrix for use in iterative algorithms on vector processors, Computing, 22 (1979), pp. 257-268.

[11] M. EIERMAnN, On semiiterative methods generated by Faber polynomials, Numer. Math., 56 (1989), pp. 139156.

[12] M. Eiermann AND W. Niethammer, On the construction of semi-iterative methods, SIAM J. Numer. Anal., 20 (1983), pp. $1153-1160$

[13] M. Eiermann, W. Niethammer, AND R. S. VARGa, A study of semi-iterative methods for nonsymmetric systems of linear equations, Numer. Math., 47 (1985), pp. 505-533.

[14] S. W. ELLACOTT, Computation of Faber series with application to numerical polynomial approximation in the complex plane, Math. Comp., 40 (1983), pp. 575-587.

[15] H. C. Elman, O. G. ERnst, AND D. P. O'LeARY, A multigrid method enhanced by Krylov subspace iteration for discrete Helmhotz equations, SIAM J. Sci. Comput., 23 (2001), pp. 1291-1315.

[16] B. ENGQUIST AND L. YING, Sweeping preconditioner for the Helmholtz equation: hierarchical matrix representation, Comm. Pure Appl. Math., 64 (2011), pp. 697-735.

[17] Y. A. ERLANGGA, Advances in iterative methods and preconditioners for the Helmholtz equation, Arch. Comput. Methods Eng., 15 (2008), pp. 37-66.

[18] Y. A. ERLANGGA AND R. NABBEN, On a multilevel Krylov method for the Helmholtz equation preconditioned by shifted Laplacian, Electron. Trans. Numer. Anal., 31 (2008), pp. 403-424. http://etna.ricam.oeaw.ac.at/vol.31.2008/pp403-424.dir/pp403-424.pdf

[19] Y. A. ERLANGGA, C. W. OOSTERLEE, AND C. VUIK, A novel multigrid based preconditioner for heterogeneous Helmholtz problems, SIAM J. Sci. Comput., 27 (2006), pp. 1471-1492.

[20] Y. A. ERLANGGa, C. VUIK, AND C. W. OosterleE, On a class of preconditioners for solving the Helmholtz equation, Appl. Numer. Math., 50 (2004), pp. 409-425.

[21] - Comparison of multigrid and incomplete LU shifted-Laplace preconditioners for the inhomogeneous Helmholtz equation, Appl. Numer. Math., 56 (2006), pp. 648-666.

[22] O. G. ERNST AND M. J. GANDER, Why it is difficult to solve Helmholtz problems with classical iterative methods, in Numerical Analysis of Multiscale Problems, I. G. Graham, T. Y. Hou, O. Lakkis, and R. Scheichl, eds., vol. 83 of Lect. Notes Comput. Sci. Eng., Springer, Heidelberg, 2012, pp. 325-363.

[23] M. J. GANDER, I. G. GRAHAM, AND E. A. SPENCE, Applying GMRES to the Helmholtz equation with shifted Laplacian preconditioning: what is the largest shift for which wavenumber-independent convergence is guaranteed?, Numer. Math., 131 (2015), pp. 567-614.

[24] M. J. GANDER AND H. ZHANG, Optimized Schwarz methods with overlap for the Helmholtz equation, in Domain Decomposition Methods in Science and Engineering XXI, J. Erhel, M. J. Gander, L. Halpern, G. Pichot, T. Sassi, and O. Widlund, eds., vol. 98 of Lect. Notes Comput. Sci. Eng, Springer, Cham, 2014, pp. 207-215.

[25] L. GaRCÍA RAmos AND R. NABBen, On the spectrum of deflated matrices with applications to the deflated shifted Laplace preconditioner for the Helmholtz equation, SIAM J. Matrix Anal. Appl., 39 (2018), pp. 262-286.

[26] G. H. Golub and C. F. Van LoAn, Matrix Computations, 4th ed., Johns Hopkins University Press, Baltimore, 2013.

[27] I. G. GRaham, E. A. Spence, ANd E. Vainik Ko, Domain decomposition preconditioning for high-frequency Helmholtz problems with absorption, Math. Comp., 86 (2017), pp. 2089-2127.

[28] M. HASSON, Expansion of analytic functions of an operator in series of Faber polynomials, Bull. Austral. Math. Soc., 56 (1997), pp. 303-318.

[29] V. Heuveline AND M. SADKANe, Arnoldi-Faber method for large non-Hermitian eigenvalue problems, Electron. Trans. Numer. Anal., 5 (1997), pp. 62-76. http://etna.ricam.oeaw.ac.at/vol.5.1997/pp62-76.dir/pp62-76.pdf

[30] F. IhlENBURG, Finite Element Analysis of Acoustic Scattering, Springer, New York, 1998.

[31] T. Koch AND J. LIESEN, The conformal "bratwurst" maps and associated Faber polynomials, Numer. Math., 86 (2000), pp. 173-191.

[32] T. KöVAri AND C. Pommerenke, On Faber polynomials and Faber expansions, Math. Z., 99 (1967), pp. 193-206.

[33] J. Kraus, P. Vassilevski, And L. Zikatanov, Polynomial of best uniform approximation to $1 / x$ and smoothing in two-level methods, Comput. Methods Appl. Math., 12 (2012), pp. 448-468. 


\section{ETNA}

Kent State University and

Johann Radon Institute (RICAM)

[34] D. Lahaye, J. Tang, And K. VuiK, eds., Modern Solvers for Helmholtz Problems, Geosyst. Math., Birkhäuser/Springer, Cham, 2017.

[35] C. LAnCzos, Chebyshev polynomials in the solution of large-scale linear systems, in Proceedings of the Association for Computing Machinery, Toronto, 1952, J. W. Forrester and R. W. Hamming, eds., Sauls Lithograph Co., Washington D. C., 1953, pp. 124-133.

[36] J. LIESEN, Faber polynomials corresponding to rational exterior mapping functions, Constr. Approx., 17 (2001), pp. 267-274.

[37] Q. LiU, R. B. Morgan, And W. Wilcox, Polynomial preconditioned GMRES and GMRES-DR, SIAM J. Sci. Comput., 37 (2015), pp. S407-S428.

[38] I. Livshits, Use of shifted Laplacian operators for solving indefinite Helmholtz equations, Numer. Math. Theory Methods Appl., 8 (2015), pp. 136-148.

[39] T. A. MAnteuffel AND S. V. PARTER, Preconditioning and boundary conditions, SIAM J. Numer. Anal., 27 (1990), pp. 656-694.

[40] A. I. MarkUsheVich, Theory of Functions of a Complex Variable. Vol. III, Prentice-Hall, Englewood Cliffs, 1967.

[41] J. M. Melenk, On Generalized Finite-Element Methods, PhD. Thesis, Graduate School, University of Maryland, College Park, 1995.

[42] I. Moret AND P. NovATI, The computation of functions of matrices by truncated Faber series, Numer. Funct. Anal. Optim., 22 (2001), pp. 697-719.

[43] - An interpolatory approximation of the matrix exponential based on Faber polynomials, J. Comput. Appl. Math., 131 (2001), pp. 361-380.

[44] V. PAATERo, Über die konforme Abbildung von Gebieten, deren Ränder von beschränkter Drehung sind, PhD. Thesis, Helsinki, 1931.

[45] B. Reps, W. Vanroose, AND H. BIn ZubAir, On the indefinite Helmholtz equation: complex stretched absorbing boundary layers, iterative analysis, and preconditioning, J. Comput. Phys., 229 (2010), pp. 8384-8405.

[46] O. RUNBORG, Helmholtz Equation and High Frequency Approximations, Lecture Notes 5: Numerical Solutions of Differential Equations, Royal Institute of Technology, Stockholm, May 2012.

[47] Y. SAAD, Practical use of polynomial preconditionings for the conjugate gradient method, SIAM J. Sci. Statist. Comput., 6 (1985), pp. 865-881.

[48] - Iterative Methods for Sparse Linear Systems, 2nd ed., SIAM, Philadelphia, 2003.

[49] A. H. Sheikh, D. Lahaye, L. Garcia Ramos, R. Nabben, and C. Vuik, Accelerating the shifted Laplace preconditioner for the Helmholtz equation by multilevel deflation, J. Comput. Phys., 322 (2016), pp. 473-490.

[50] A. H. SHEIKH, D. LAHAYE, AND C. VUIK, On the convergence of shifted Laplace preconditioner combined with multilevel deflation, Numer. Linear Algebra Appl., 20 (2013), pp. 645-662.

[51] V. I. Smirnov And N. A. Lebedev, Functions of a Complex Variable: Constructive Theory, M.I.T. Press, Cambridge, 1968.

[52] G. STARKE AND R. S. VARGA, A hybrid Arnoldi-Faber iterative method for nonsymmetric systems of linear equations, Numer. Math., 64 (1993), pp. 213-240.

[53] C. C. Stolk, A rapidly converging domain decomposition method for the Helmholtz equation, J. Comput. Phys., 241 (2013), pp. 240-252.

[54] P. K. Suetin, Series of Faber Polynomials, vol. 1 of Analytical Methods and Special Functions, Gordon and Breach Science, Amsterdam, 1998.

[55] M. B. VAN GIJZEN, A polynomial preconditioner for the GMRES algorithm, J. Comput. Appl. Math., 59 (1995), pp. 91-107.

[56] M. B. VAN GIJZEN, Y. A. ERLANGGA, AND C. VUIK, Spectral analysis of the discrete Helmholtz operator preconditioned with a shifted Laplacian, SIAM J. Sci. Comput., 29 (2007), pp. 1942-1958.

[57] R. S. VARGA, Matrix Iterative Analysis, expanded ed., Springer, Berlin, 2000.

[58] Y. XI AND Y. SAAD, A rational function preconditioner for indefinite sparse linear systems, SIAM J. Sci. Comput., 39 (2017), pp. A1145-A1167.

[59] L. ZEPEDA-NÚÑEZ AND L. DEMANET, The method of polarized traces for the 2 D Helmholtz equation, J. Comput. Phys., 308 (2016), pp. 347-388. 